\title{
Myeloid loss of Beclin 1 promotes PD-L1hi precursor B cell lymphoma development
}

\author{
Peng Tan, ${ }^{1,2}$ Lian He, ${ }^{2}$ Changsheng Xing, ${ }^{1}$ lingrong Mao, ${ }^{1,3}$ Xiao Yu, ${ }^{1}$ Motao Zhu, ${ }^{1}$ Lixia Diao, ${ }^{4}$ Leng Han, ${ }^{5}$ Yubin Zhou, ${ }^{2}$ \\ M. James You, ${ }^{6}$ Helen Y. Wang, ${ }^{1}$ and Rong-Fu Wang ${ }^{1,2,7}$ \\ 'Center for Inflammation and Epigenetics, Houston Methodist Research Institute, Houston, Texas, USA. Institute of Biosciences and Technology, College of Medicine, Texas A\&M University, Houston, \\ Texas, USA. ${ }^{3}$ Xiangya Hospital, Central South University, Changsha, China. ${ }^{4}$ Department of Bioinformatics and Computational Biology, The University of Texas MD Anderson Cancer Center, Houston, \\ Texas, USA. ${ }^{5}$ Department of Biochemistry and Molecular Biology, The University of Texas Health Science Center at Houston McGovern Medical School, Houston, Texas, USA. ${ }^{6}$ Department of Hematopathology, \\ The University of Texas MD Anderson Cancer Center, Houston, Texas, USA. `Department of Microbiology and Immunology, Weill Cornell Medical College, Cornell University, New York, New York, USA.
}

\begin{abstract}
Beclin 1 (Becn1) is a key molecule in the autophagy pathway and has been implicated in cancer development. Due to the embryonic lethality of homozygous Becn1-deficient mice, the precise mechanisms and cell type-specific roles of Becn1 in regulating inflammation and cancer immunity remain elusive. Here, we report that myeloid-deficient Becn1 (Becn1 ${ }^{\mathrm{MM})}$ mice developed neutrophilia, were hypersusceptible to LPS-induced septic shock, and had a high risk of developing spontaneous precursor B cell (pre-B cell) lymphoma with elevated expression of immunosuppressive molecules programmed death ligand 1 (PD-L1) and IL-10. Becn1 deficiency resulted in the stabilization of MEKK3 and aberrant p38 activation in neutrophils, and mediated neutrophil-B cell interaction through Cxc19/Cxcr3 chemotaxis. Neutrophil-B cell interplay further led to the activation of IL-21/STAT3/IRF1 and CD40L/ERK signaling and PD-L1 expression; therefore, it suppressed CD8 ${ }^{+}$cell function. Ablation of p38 in Becn $1^{\lrcorner M}$ mice prevented neutrophil inflammation and B cell tumorigenesis. Importantly, the low expression of Becn1 in human neutrophils was significantly correlated with the PD-L1 levels in pre-B acute lymphoblastic lymphoma (ALL) patients. Our findings have identified myeloid Becn1 as a key regulator of cancer immunity and therapeutic target for pre-B cell lymphomas.
\end{abstract}

\section{Introduction}

Autophagy has been well recognized as a key intracellular degradation and recycling machinery for maintaining cellular homeostasis, whose dysregulation leads to cancer (1). Beclin 1 (Becn1) acts as a core component of the class III phosphatidylinositol 3-kinase Vps34 complex, which is critical for the initiation of autophagy and autophagosome formation (2). Becn1 was first described as a tumor suppressor, as monoallelic deletion of Becn1 results in spontaneous tumor formation (3). However, given the complexity of the tumor microenvironment, which requires spatiotemporal interactions between tumor cells and other nontumor components, such as fibroblasts and stroma, endothelial, and myeloid cells, the cell type-specific roles of Becn1 in tumor development and immunity are still elusive. Neutrophils are an important component in cancer immunity (4) and regulate many other diseases, such as Alzheimer's disease (5), through the release of cytokines IL-21 and IL-17 and neutrophil extracellular traps (NETs). For instance, neutrophils with B cell helper phenotype are correlated with tumor development (6-8). However, the detailed mechanism

\section{Related Commentary: p. 5079}

Conflict of interest: The authors have declared that no conflict of interest exists. Copyright: (5) 2019, American Society for Clinical Investigation.

Submitted: January 25, 2019; Accepted: August 29, 2019; Published: October 28, 2019. Reference information: J Clin Invest. 2019;129(12):5261-5277.

https://doi.org/10.1172/JCI127721. that controls the neutrophil differentiation toward a protumorigenic B cell helper phenotype is undefined.

Cancer cells escape from $\mathrm{T}$ cell-mediated cytotoxicity by exploiting the inhibitory immune checkpoint molecules, including the receptor of programmed death 1 (PD-1) and its ligand PD-L1. Binding of PD-L1 to its receptor, PD-1, on activated T cells inhibits the T cell-activating signals and antitumor immunity (9). Notably, B cell lymphomas also leverage the PD-L1/PD-1 checkpoint to induce immune escape (10). PD-L1 expression in cancer cells is regulated by mechanisms that include aberrant oncogenic and inflammatory signaling and protein stability (9). However, mechanisms regarding the recruitment of specific myeloid subsets to interact with cancer cells and drive the tumor development and immune evasion through immune checkpoint molecules are still elusive.

In this study, we show that neutrophil-derived inflammation is critical for mouse survival in LPS-induced septic shock and responsible for the high incidence $(\sim 25 \%)$ of spontaneous precursor B cell (pre-B cell) lymphoma development in mice with myeloid-specific ablation of Becn1. We further identify Becn1 as a neutrophil-specific negative regulator of the MEKK3/p38 signaling axis in an autophagy-independent manner, which is critical for (a) expression of B cell prosurvival factors IL-17, IL-21, and B cell-activating factor (BAFF); (b) interaction of neutrophils and B cells through CXCR3/CXCL9 chemotaxis; and (c) persistent activation of IL-21/STAT3-IRF1 and CD40L/ERK signaling in B cells that together regulate PD-L1 expression to suppress antitumor $\mathrm{CD}^{+} \mathrm{T}$ cell function. Consistently, by analyzing the human 
pre-B cell acute lymphoblastic lymphoma (ALL) patient samples, we reveal that the low expression of Becn1 in tumor-infiltrated neutrophils correlates with tumor PD-L1 expression, and similar positive correlations are also observed between any 2 of the following 3 factors: neutrophil marker, IL-21, and PD-L1. Together, our findings identify myeloid Becn 1 as a non-cell-autonomous tumor suppressor in the regulation of pre-B cell malignancy and immunosuppressive function.

\section{Results}

Myeloid-specific ablation of Becn1 induces splenomegaly and lymphadenopathy with neutrophilia. To investigate the role of Becn1 in myeloid lineage development and innate immune response, we crossed $B e c n f^{f l}$ mice with mice expressing the lysozyme promoterdriven Cre recombinase gene (Lyz2-Cre), designated as Becn1 ${ }^{1 M}$ mice. Cohoused littermate Becn1 ${ }^{\mathrm{WT}}$ or Becn1 $1^{\text {flox }}$;Lyz2-Cre ${ }^{-}$mice were used as WT controls. Conditional Becn1 deletions in $\mathrm{F} 4 / 80^{+} \mathrm{CD}^{-} 1 \mathrm{~b}^{+}$ peritoneal macrophages (pMAC) and $\mathrm{Ly} 6 \mathrm{G}^{+} \mathrm{CD} 11 \mathrm{~b}^{+}$neutrophils were verified by immunoblot analyses compared with $\mathrm{CD} 11 \mathrm{c}^{+}$conventional DCs (cDCs), CD4 ${ }^{+}$T cells, and CD19 ${ }^{+} \mathrm{B}$ cells (Supplemental Figure 1A; supplemental material available online with this article; https://doi.org/10.1172/JCI127721DS1). Notably, Becn1 ${ }^{4 M}$ mice developed splenomegaly and had profound enlargements on inguinal, axillary, and mesenteric LNs (Figure 1, A and B). Spleen (SP) mass and the total number of splenocytes were markedly increased (Figure 1A and Supplemental Figure 1B). Histological analysis of SP sections revealed a marked effacement of the splenic architecture, with loss of marginal zone (MZ) barriers and a concomitant loss of the clear-cut delimitation between the lymphoid and myeloid parenchyma (Figure 1A). Hematologic analysis of peripheral blood in Becn $1^{\Delta M}$ mice showed increased circulating neutrophils, white blood cells, and eosinophils, but decreased platelets (Supplemental Table 1). Consistently, expanded Ly6 $\mathrm{G}^{+}$splenic neutrophils with normal shape and segmentation accumulated in the red pulp of SP surrounding MZ B cells (Supplemental Figure 1C).

Loss of myeloid-specific Becn1 markedly increased the number of $\mathrm{Gr}-1^{+} \mathrm{CD} 11 \mathrm{~b}^{+}$myeloid-lineage cells, which is mainly contributed to by the increase of $\mathrm{Ly} 6 \mathrm{G}^{+} \mathrm{CD} 11 \mathrm{~b}^{+}$neutrophils in the $\mathrm{BM}$ and SP. However, the percentages of $\mathrm{F} 4 / 80^{+} \mathrm{CD}_{11 \mathrm{~b}^{+}}$macrophages and $\mathrm{CD} 11 \mathrm{~b}^{+} \mathrm{Ly} 6 \mathrm{C}^{+}$monocytic cells remained unchanged (Figure 1, C-G). Besides, we found an increase in total SP B220 $\mathrm{B}$ cells in some of the Becn $1^{\Delta M}$ mice (Figure $1 \mathrm{C}$ ), but not $\mathrm{B} 22 \mathrm{O}^{+} \mathrm{CD}$ $11 \mathrm{c}^{+} \mathrm{CD} 11 \mathrm{~b}^{-}$plasmacytoid DCs (pDCs) (Supplemental Figure 1D). There was no significant difference in the percentages of different $\mathrm{CD}^{+} \mathrm{T}$ cell subsets and $\mathrm{T}$ cell functions for cytokine release (Supplemental Figure 1, E and F). Gr- $1^{+} \mathrm{CD} 11 \mathrm{~b}^{+}$cells and F4/80 ${ }^{+} \mathrm{CD} 11 \mathrm{~b}^{+}$ cells also showed equal levels of apoptosis (Supplemental Figure $1 G)$. We further transplanted a 1:1 mixture of CD45.1 $1^{+} \mathrm{WT}$ and Becn $1^{4 M}$ CD 45.2 $2^{+}$BMs into lethally irradiated CD 45.1 $1^{+}$WT mice, in which Becn1 ${ }^{4 M} \mathrm{CD} 45.2^{+} \mathrm{BM}$ cells showed a growth advantage (Supplemental Figure $1 \mathrm{H}$ ). The neutrophil counts of mice reconstituted with Becn $1^{4 M} \mathrm{CD} 45.2^{+} \mathrm{BM}$ cells were approximately 4-fold higher than those that received CD $45.2^{+}$WT BM (Supplemental Figure 1I). Together, our results suggest that neutrophilia in $B e c n 1^{\Delta M}$ mice is driven by factors intrinsic to hematopoietic cells, causing accelerated proliferation and/or enhanced survival of neutrophil progenitors or mature neutrophils.
Becn1 negatively regulates $p 38$ activation and inflammation in neutrophils. We next examined TLR-mediated innate immune signaling responses between WT and Becn1-deficient neutrophils and macrophages in the periphery of 6-to 8-week-old mice and found that p38 MAPK activity was increased, while inhibitor of $\mathrm{NF}-\kappa \mathrm{B}$ (IКB) kinase (IKK) activation was slightly reduced in neutrophils after LPS (a TLR4 ligand) or Pam3CSK4 (a TLR2 ligand) stimulation (Figure 2, A and B, and Supplemental Figure 2, A and B). Becn1-deficient neutrophils produced a higher amount of TNF- $\alpha$ and IL-1 $\beta$, but Becn1-deficient macrophages produced higher amounts of TNF- $\alpha$ and IL-6 (Figure 2C). Consistently, we observed an increase in the expression of pro-IL-1 $\beta$ in Becn1deficient neutrophils, but not in macrophages (Figure 2D). It has been shown that there was significant accumulation of neutrophils in the model of Listeria-infected $S R B 1^{-/-}$mice compared with WT mice, suggesting a critical role of SR-B1 in the differential regulation of neutrophils and macrophages (11). However, no difference was observed in Scarb1 expression in Becn1-deficient neutrophils and macrophages with or without LPS treatment (Supplemental Figure $2 \mathrm{C}$ ). Therefore, the differential regulation of Becn 1 in the activation of $\mathrm{p} 38$ and proinflammatory cytokine production is cell type specific, but independent of SR-B1.

Also, Becn1 expression was induced during M2 macrophage polarization by IL-4 or IL-10 (Supplemental Figure 2D). Becn1deficient BM macrophages were readily differentiated into proinflammatory M1 macrophages by LPS (Supplemental Figure 2E). To further define the physiological function of Becn1, we showed that Becn $1^{\Delta M}$ mice died after LPS-induced endotoxin shock (30 $\mathrm{mg} / \mathrm{kg}$ ) within 12 hours compared with WT mice, which survived for up to 40 hours (Figure 2E). Consistent with this observation, $B e c n 1^{1 M}$ mice had markedly elevated serum concentrations of proinflammatory cytokines such as TNF- $\alpha$, IL-6, and IL-1 $\beta$ after LPS treatment (Figure 2F). Since ROS are closely linked to inflammation (12), we treated neutrophils and macrophages with LPS for 180 minutes and then incubated cells with CM-H2DCFDA for 30 minutes. Flow cytometry analysis revealed a modest increase of ROS production in Becn1-deficient neutrophils (Supplemental Figure 2F). In contrast, ROS production was lower in Becn1-deficient macrophages than in WT cells after LPS stimulation (Supplemental Figure 2F). To determine which cell type contributed to the susceptibility of Becn1 $1^{4 M}$ mice to LPS treatment, we depleted macrophages using clodronate-containing liposomes (13) and neutrophils using Ly6G antibody (IA8) (ref. 14 and Supplemental Figure $2 \mathrm{G}$ ). We found that macrophage depletion did not change mouse survival after LPS-induced septic shock. However, there was significantly prolonged survival in neutrophil-depleted mice (Figure $2 \mathrm{G}$ ), indicating that neutrophils play a dominant role in LPS-induced septic shock. These results suggest that Becn1 deletion in myeloid cells enhances the sensitivity and severity of LPS-induced septic shock, probably through the increased serum concentrations of proinflammatory cytokines.

To determine the role of Becn1 in regulating innate immune signaling and gene expression in neutrophils, we performed RNA-Seq analysis of freshly isolated neutrophils after 4 hours of LPS treatment. Interestingly, we observed marked upregulation of genes involved in pathways related to inflammation, rheumatoid arthritis, and inflammatory bowel diseases (Figure 
A
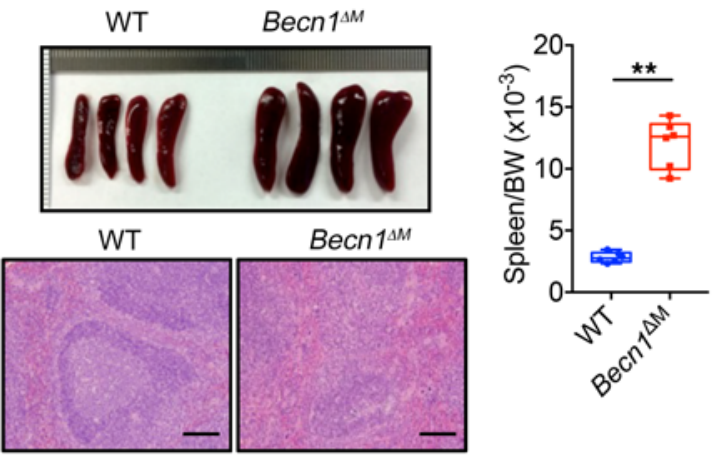

B

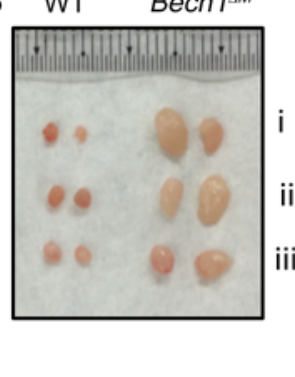

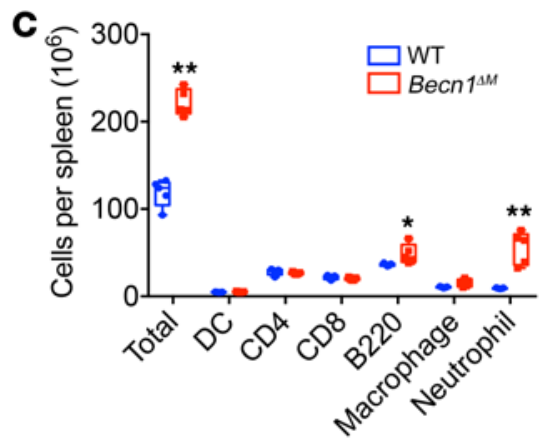

D

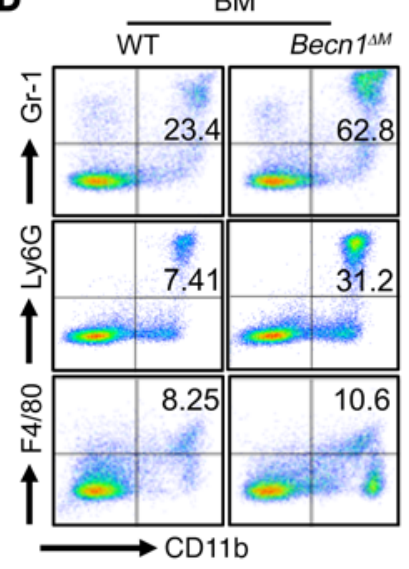

$\mathbf{F}$

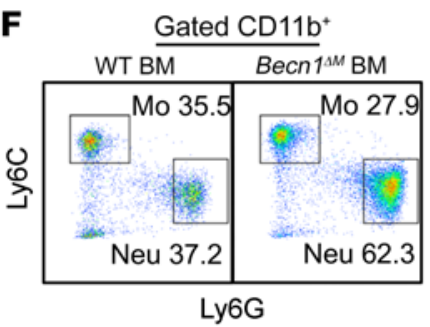

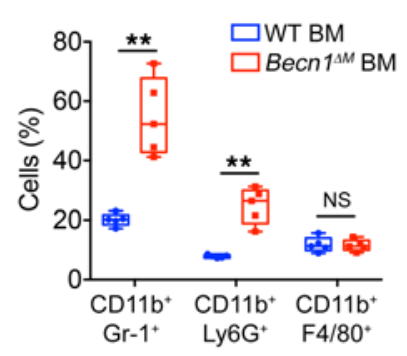

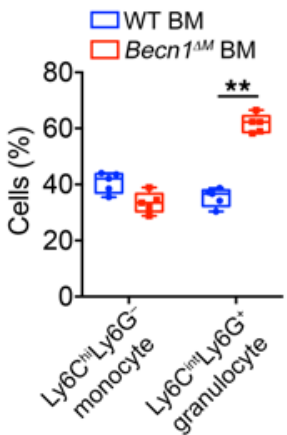

$\mathbf{E}$
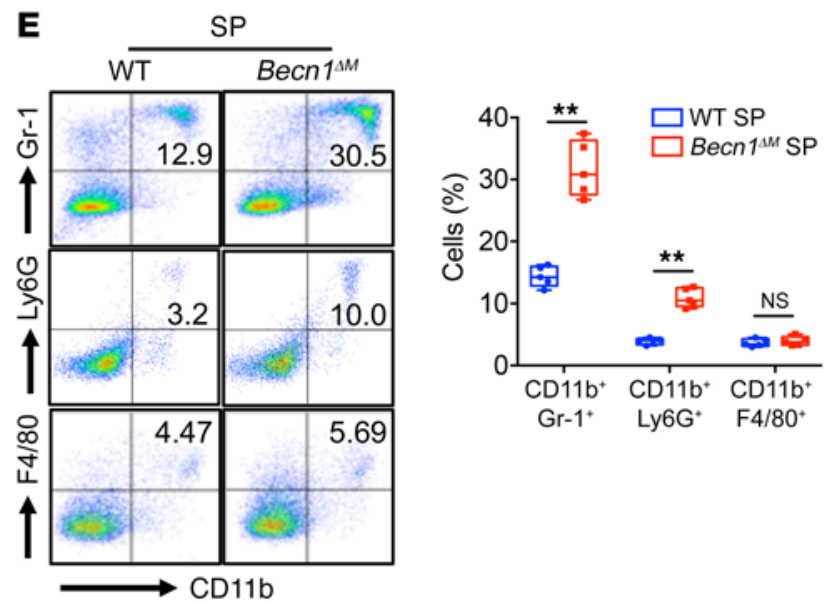

Figure 1. Characterization and phenotypic analysis of Becn $1^{\mathrm{MM}}$ mice. (A) SP size comparison between WT and Becn $1^{1 \mathrm{M}}$ mice and SP/ body weight ratio $(n=$ 4). H\&E staining of SP sections from WT and Becn $7^{4 M}$ mice. Scale bars: $500 \mu \mathrm{m}$. (B) Lymphadenopathy in Becn $7^{1 M}$ mice compared with WT control. Inguinal (i), axillary (ii), and mesenteric (iii) LNs were examined. Data are representative of 3 independent experiments with 6 - to 8 -week-old mice ( $n=2$ ) in each

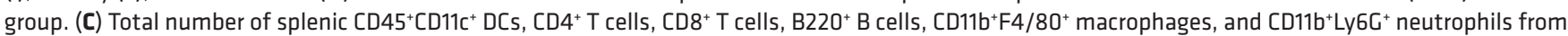

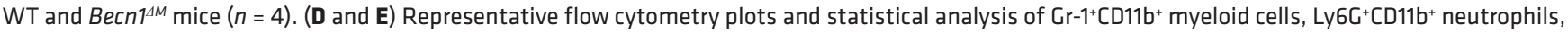
and $\mathrm{F} 4 / 80^{+} \mathrm{CD} 11 \mathrm{~b}^{+}$macrophages in BM (D) and SP (E) of WT and Becn ${ }^{\mathrm{MM}}$ mice $(n=5)$. (F and $\left.\mathbf{G}\right)$ Representative flow cytometry plots and statistical analysis of monocytic (Ly6C ${ }^{\text {hi }}$ Ly6C $^{-}$) and granulocytic (Ly6C int Ly6C ${ }^{+}$) cells in BM (F) and SP (G) of 6-to 8-week-old WT and Becn $7^{1 M}$ mice $(n=5)$. Data represented in $\mathbf{A}$ and C-G were from 6- to 8-week-old mice and are presented as box plots, with lines representing median and error bars showing mean \pm SEM. Statistical differences between groups were calculated using Student's unpaired $t$ test. ${ }^{*} P<0.05 ;{ }^{*} P<0.01$.

$2 \mathrm{H}$, Supplemental Figure $2 \mathrm{H}$, and Supplemental Table 2). The key candidates included genes encoding IL-17 and related cytokines, chemokine receptor and chemotaxis, cell metabolism, and immunity. Neutrophils are another source of IL-17 besides Th17 cells $(5,15-17)$. We next isolated Becn1-deficient neutrophils and pMACs, treated them with LPS, and found significantly higher levels of IL-17A and IL-21 in Becn1-deficient neutrophils compared with WT controls or macrophages (Supplemental Figure 2I). Together, our results suggest that Becn1 deletion enhanc- es p38 activation and produces large amounts of IL-1 $1 \beta$, TNF- $\alpha$, IL-17, and IL-21 in neutrophils.

Autophagy-independent degradation of MEKK3 by Becn1 suppresses p38 signaling. Since NF- $\mathrm{B}$ and MAPK pathways trigger the downstream signaling of IKK complex, Erk1/2, p38, and JNK through the activation of MAP3Ks (TAK1, ASK1, MEKK3) (18), we tested to determine whether Becn1 interacted with these key signaling molecules and observed strong interaction of Becn1 with MEKK3 and MKK3, only weak interaction with IKK $\beta$ and NEMO, 


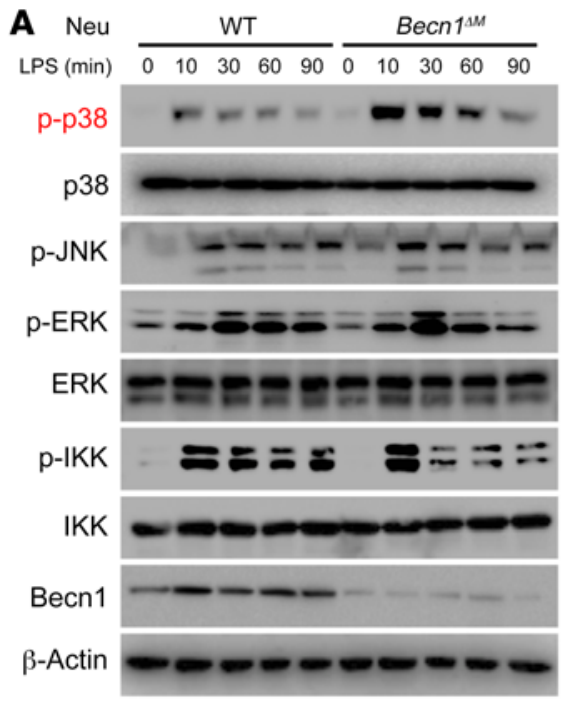

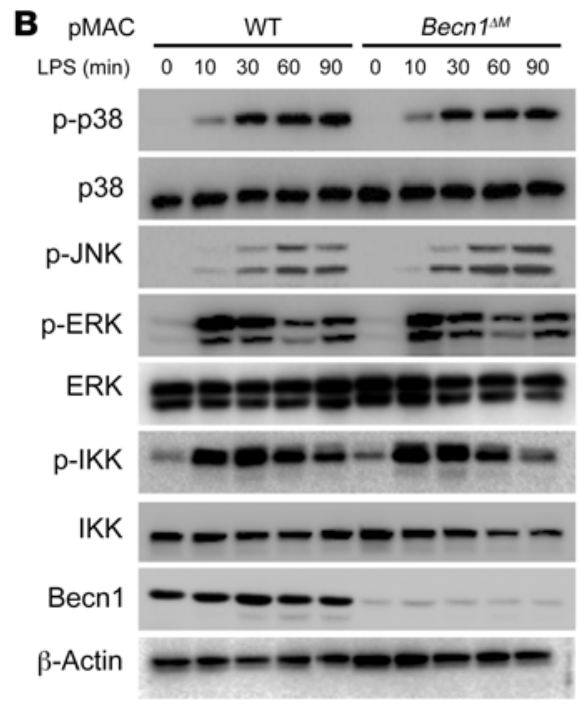
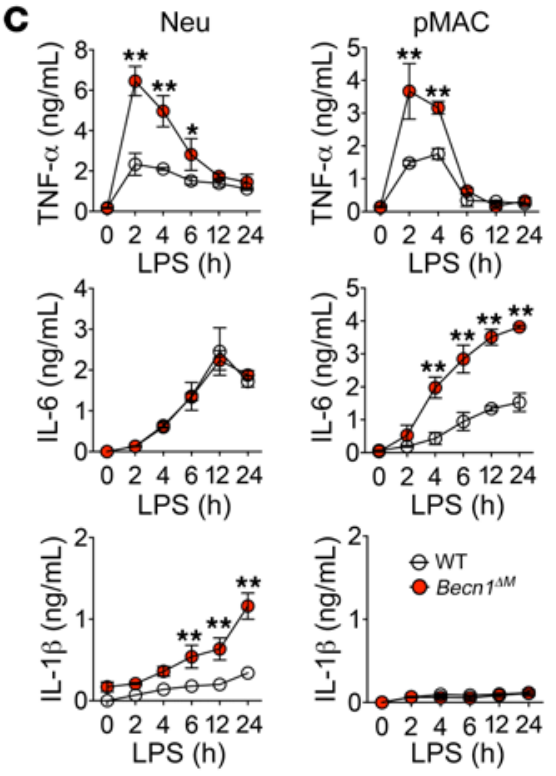

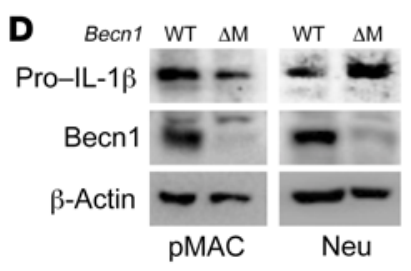

G
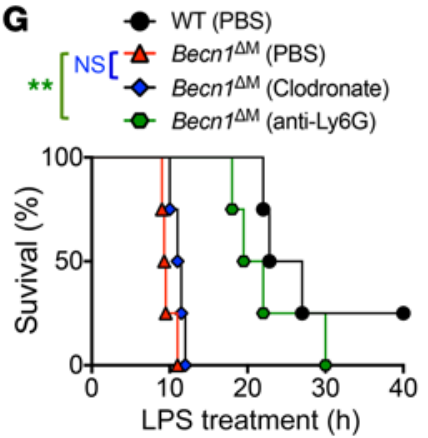
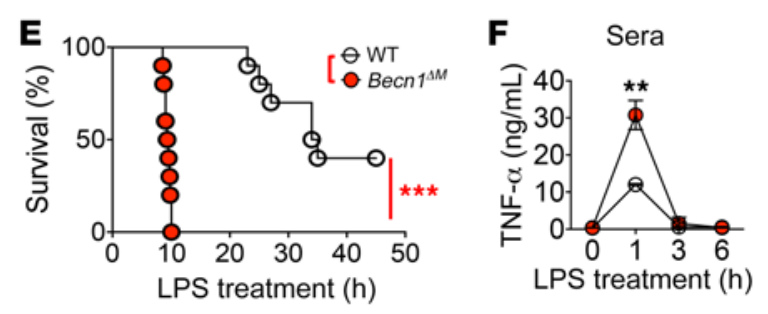
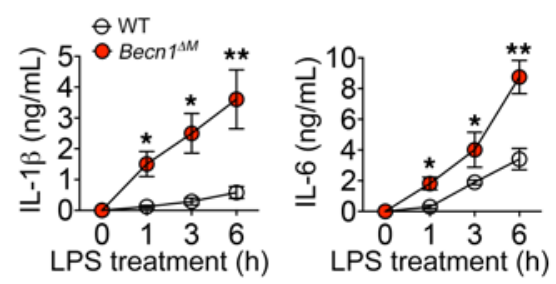

H

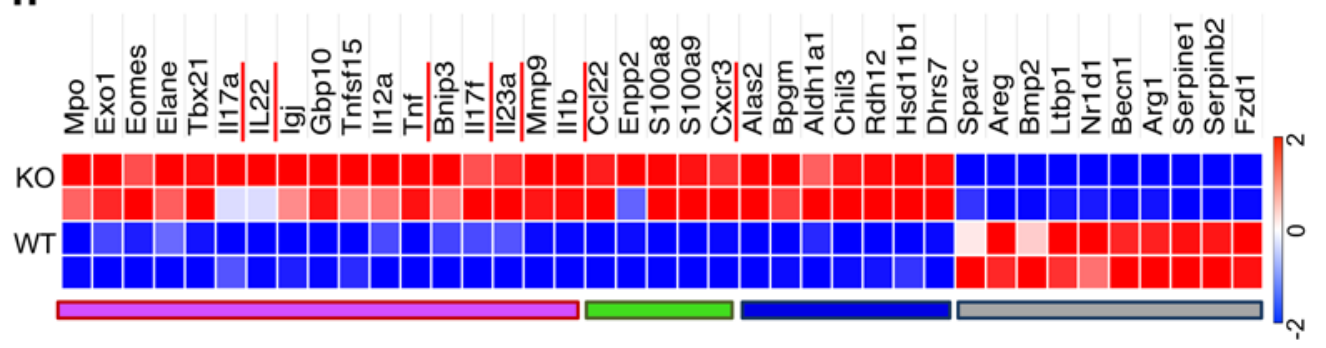

Figure 2. Cell type-specific regulation of Becn1 in proinflammatory signaling pathways and immune responses. (A and B) Neu (A) or pMAC (B) from the periphery of WT and Becn $7^{1 M}$ mice were treated with LPS for the indicated time points, followed by IB with indicated antibodies. (C) ELISA measurement of TNF- $\alpha$, IL-6, and IL-1 $\beta$ production by Neu and pMAC of WT and Becn $7^{1 \mathrm{M}}$ mice treated with $100 \mathrm{ng} / \mathrm{ml}$ LPS for indicated time points ( $\left.n=4\right)$. (D) IB of proIL-1 $\beta$ expression in Neu or PMAC from WT and Becn $1^{1 M}$ mice. (E) Survival of WT and Becn $1^{\mathrm{MM}}$ mice $(n=10$; female) treated with high-dose LPS (30 mg/kg, i.p.). (F) Plasma concentrations of TNF- $\alpha$, IL-6, and IL-1 $\beta$ in WT or Becn1 ${ }^{1 M}$ mice $(n=5)$ at indicated time points after LPS treatment. (G) Survival of WT and Becn $7^{M M}$ mice ( $n=4$; female) treated with PBS- or clodronate-containing liposomes to deplete macrophages or with anti-Ly6C antibody (1A8) to deplete neutrophils, followed by high-dose LPS treatment. (H) Heatmap representation of differential expressed genes in neutrophils isolated from Becn $7^{1 \mathrm{M}}$ mice compared with WT controls: neutrophil-mediated immunity and IL-17-related cytokines (purple), chemokine receptor and neutrophil-chemotaxis (green), cell metabolism (blue). Data shown in A, B, and $\mathbf{D}$ are representative of 3 independent experiments with 6 - to 8-week-old mice ( $n=3$; female) in each group. Statistical differences between groups were calculated using Student's unpaired $t$ test (mean \pm SEM) (C and F) and Mantel-Cox log-rank test (E and G). ${ }^{*} P<0.05 ;{ }^{* *} P<0.01 ;{ }^{* *} P<0.001$.

and no interaction with IKK $\alpha$, ASK1, TAK1, or p38 (Figure 3, A and B). Importantly, we found that MEKK3 protein was markedly reduced with an increasing amount of Becn1 in 293T cells (Figure 3C). Consistently, we found that the endogenous level of MEKK3 was markedly increased in Becn1-deficient neutrophils, but not macrophages (Figure 3, D and E). In addition, Becn1 deficiency increased the activation of MKK3/6, the MEKK3-downstream mediator for p38 activation (Figure $3 \mathrm{~F}$ ). These results suggest that Becn1 regulates MEKK3 stability and MEKK3/p38 signaling activation in neutrophils.

To understand how Becn1 regulates MEKK3 for degradation, we showed that proteasome inhibitor MG132, but not autophagy inhibitors 3-methyladenine (3-MA) or chloroquine (CQ), blocked Becn1-mediated MEKK3 degradation (Figure 3G), suggesting that Becn1 degrades MEKK3 through a proteasome-dependent manner. Protein lysine (K) 48-linked ubiquitination has emerged as an 
A

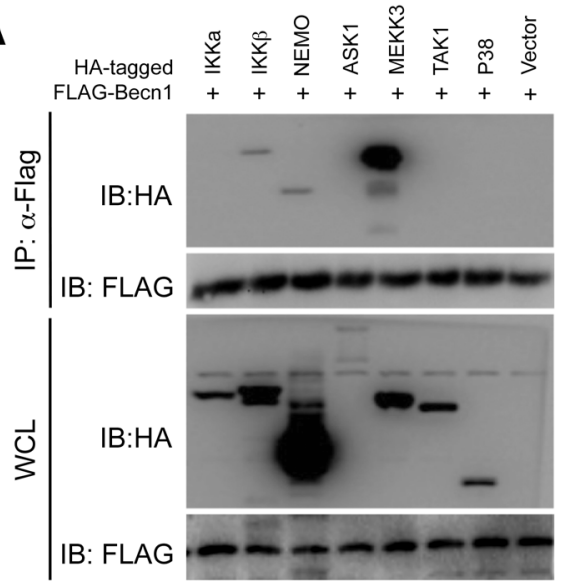

E

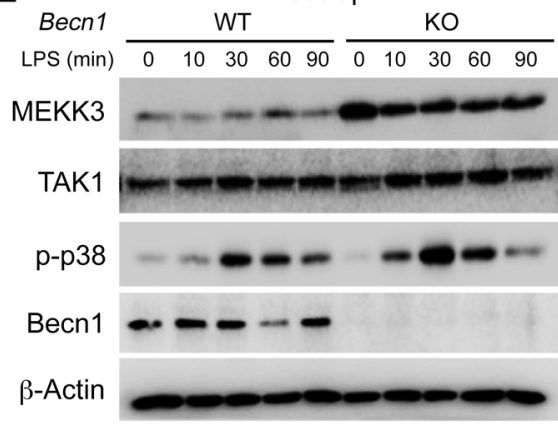

B

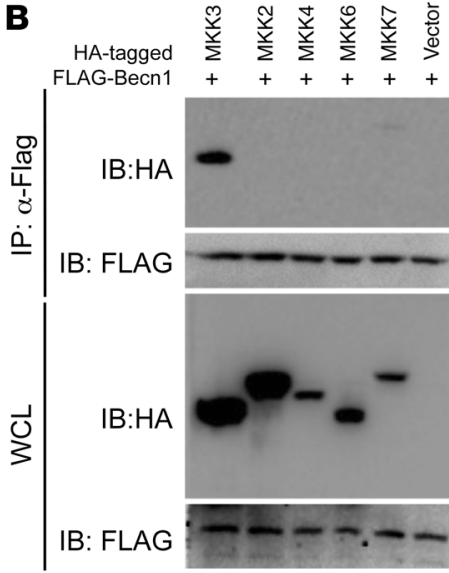

$\mathbf{F}$

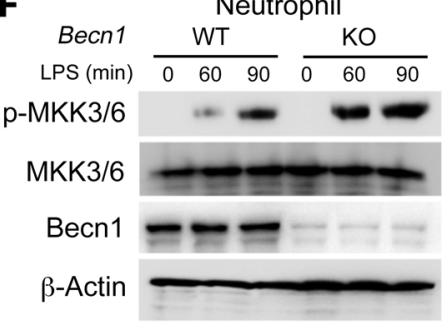

C

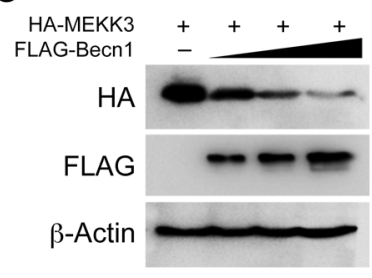

D

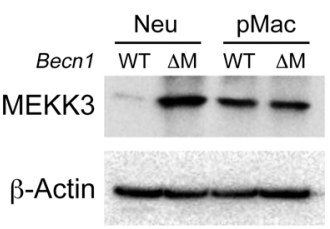

G

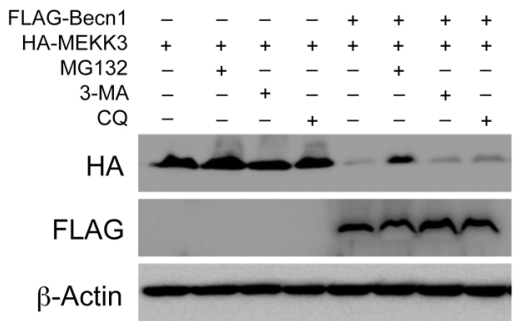

H

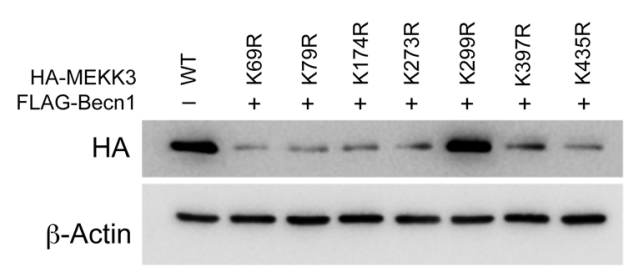

I

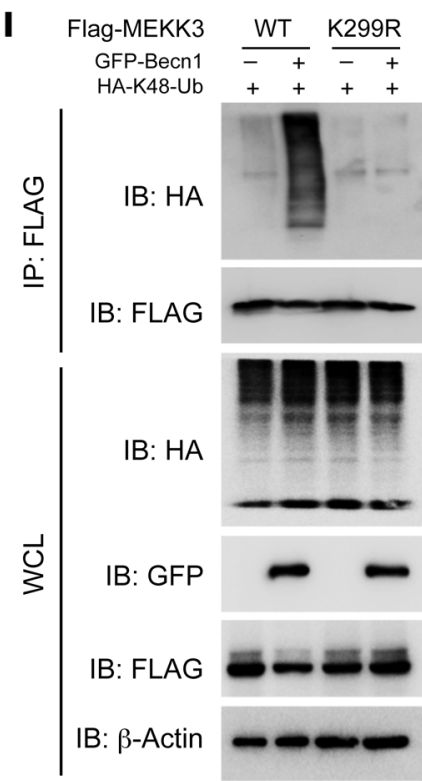

Figure 3. Becn1 ablation suppresses proteasomal degradation of neutrophil MEKK3 upstream of p38. (A and B) Screening of Becn1 binding proteins in NF-KB and MAPK pathways in 293T cells. WCL, whole-cell lysate. (C) $293 \mathrm{~T}$ cells transfected with $100 \mathrm{ng} \mathrm{HA-MEKK3}$ along with increased amounts (0, 100, 250, and 500 ng) of FLAG-Becn1, followed by IB with indicated antibodies. (D) IB of MEKK3 protein expression in Becn1-deficient Neu and pMAC compared with WT controls. (E and F) WT or Becn1-deficient neutrophils were treated with LPS for the indicated time points, followed by IB with antibodies against MEKK3, TAK1 or p-p38 (E), and MKK3/6 signaling (F). (G) IB of 293T cells transfected with HA-MEKK3 along with empty vector or FLAG-Becn1 left untreated or treated with proteasome inhibitor MG132 $(1 \mu \mathrm{M})$ or autophagy inhibitors 3-MA (5 mM) or CQ $(10 \mu \mathrm{M})$. (H) IB of $293 \mathrm{~T}$ cells transfected with WT or HAMEKK3 (K69R, K79R, K174R, K273R, K299R, K397R, K435R) along with empty vector or FLAG-Becn1. (I) 293T cells were transfected with FLAG-MEKK3 WT or FLAG-MEKK3 K299R along with GFP-Becn1 and HA-ubiquitin K48 expression vectors, followed by immunoprecipitation with FLAG beads and IB with indicated antibodies. Data are representative of 3 independent experiments in 293 T cells (A-C and G-I) and with 6- to 8-week old mice ( $n=3$; female) (D-F).

important mechanism for targeting a protein for proteasomal degradation (19). To identify key lysine residues in MEKK3 required for K48-linked ubiquitination, we substituted a series of lysine residues with arginine and found that lysine 299 (K299) residue was essential in Becn1-mediated MEKK3 ubiquitination and proteasome degradation (Figure 3, $\mathrm{H}$ and I). These results suggest that Becn1 targets MEKK3 for ubiquitination-dependent proteasomal degradation through the K299 residue in MEKK3.

Genetic ablation of $p 38$ or MEKK 3 rescues Becn $1^{1 M}$ mouse phenotypes. To determine whether specific deletion of $\mathrm{p} 38$ (encoded by Mapk14) or MEKK3 (encoded by Map3k3) could rescue the phenotypes and survival in response to LPS-induced septic shock

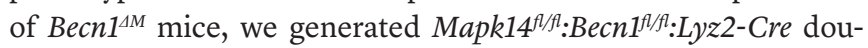
ble-KO (DKO) (Becn1 $\left.{ }^{1 M}: M a p k 14^{\lrcorner M}\right)$ mice. Gross phenotypic analysis revealed reduced SP size of Becn1 $1^{M M}: M a p k 14^{M M}$ that was comparable to that of WT SP (Figure 4A). Consistently, the percentages ( $41.4 \%$ in BM, $14.5 \%$ in SP) of CD11b $\mathrm{Gr}-1^{+}$cells in Becn $1^{1 M}$ mice were reduced to $22.2 \%$ in BM and $7.26 \%$ in SP in Becn1 $1^{4 M}:$ Mapk14 $4^{4 M}$ mice, which were similar to the levels in WT mice (Figure 4B). The serum levels of TNF- $\alpha$, IL-1 $1 \beta$, and IL-17 cytokines in Becn $1^{1 M}$ : $M a p k 14^{4 M}$ mice were also reduced to levels similar to those of WT controls, even though IL-6 production remained at a relatively 
higher level in both Becn1 $1^{4 M}: M a p k 14^{4 M}$ and Becn1 $1^{4 M}$ mice (Figure $4 \mathrm{C}$ ). Importantly, p38 ablation in Becn1 ${ }^{\Delta M}$ prolonged the survival to a level similar to that of WT mice in response to LPS-induced septic shock, but IL-6 ablation in Becn $1^{\Delta M}$ mice failed to do so (Figure $4 \mathrm{D})$, suggesting that TNF- $\alpha$ and IL-1 $\beta$, but not IL- 6 , are responsible for sensitivity to LPS-induced septic shock.

Because mouse Becn1 and Map3k3 are on the same chromosome (mm11, NC_000077.6), it is difficult to generate Map3k $3^{f / f l}$ : $B e c n 1^{f l f f}:$ Lyz2-Cre DKO mice. For this reason, we used a CRISPRCas9 genome editing approach (where CRISPR indicates clustered regularly interspaced short palindromic repeats) (20) to generate Becn1 ${ }^{1 M}: M a p 3 k 3^{\text {Cas } 9} \mathrm{BM}$ chimera. BM chimera with WT $\mathrm{BM}$ or Becn $1^{4 M} \mathrm{BM}$ transduced with nontargeting control sgRNA were used as WT or Becn $1^{1 M}$ single-KO controls. The LPS-induced phosphorylation of $\mathrm{p} 38$ in neutrophils isolated from Becn1 ${ }^{4 M}: M a p-$ $3 k 3^{\text {Cas } 9}$ mice was significantly reduced (Figure $4 \mathrm{E}$ ). In addition, neutrophil number and serum levels of proinflammatory cytokines TNF- $\alpha$, IL-1 $\beta$, and IL-17 in Becn1 ${ }^{1 M}: M a p 3 k 3^{\text {Cas } 9}$ mice were restored to WT chimeric control (Figure 4, F and G). Consistently, $B e c n 1^{4 M}: M a p 3 k 3^{\operatorname{Cas} 9}$ mice were more resistant to LPS when compared with the Becn $1^{4 M}$ group (Figure $4 \mathrm{H}$ ). These results indicate that p38 or MEKK3 ablation in neutrophils restores inflammatory cytokine (TNF- $\alpha$, IL-1 $\beta$, and IL-17) production and reduces hypersensitivity to LPS-induced septic shock observed in Becn $1^{1 M}$ mice.

Spontaneous development of metastatic pre-B cell lymphoma in $B e c n 1^{\Delta M}$ mice. During this work, we unexpectedly observed the occurrence of highly proliferative lymphoma in the cervical LNs in $40 \%$ of Becn1 $1^{\Delta M}$ mice (Figure 5 , A and B), with accumulating infiltration of tumor cells in other tissues, such as lungs, heart, and thymus (Supplemental Figure 3A). H\&E staining of the infiltrated tumor cells revealed that the medium-size tumor cells contained centrally located and prominent nucleoli (Supplemental Figure $3 \mathrm{~A})$, suggesting a pre-B cell lymphoblastic lymphoma phenotype (21). These tumor cells were positive for B220 staining, but negative for monocyte (Mac-3), macrophage (Mac-1), neutrophil (Ly6G), or T cell (CD3) markers (Figure 5C, and Supplemental Figure $3, \mathrm{~B}-\mathrm{D})$. The $\mathrm{B}$ cell origination of these tumor cells was further confirmed by PAX5 staining in lung infiltrates. Of note, lung-infiltrated tumor cells contained a majority of $\mathrm{PAX} 5^{+} \mathrm{B} 22 \mathrm{O}^{\mathrm{lo} /-}$ cells, but also $\mathrm{PAX} 5^{+} \mathrm{B}_{22} 2 \mathrm{O}^{+}$populations (Figure 5D), when using low B220 antibody concentration ( $\mathrm{T} 1,1: 500$ dilution, vs. high concentration [T2], 1:50 dilution), suggesting the heterogeneity of tumor cells. Unlike the B cell lymphoma in Becn1 ${ }^{+/-}$mice that expresses BCL-6 protein (3), we found that the $\mathrm{LN}$ or lung-infiltrated $\mathrm{B} 22 \mathrm{O}^{+}$tumor cells in Becn $1^{4 M}$ mice were negative for BCL-6 (Figure 5E).

Using CFSE labeling, we showed that B cells isolated from $B e c n 1^{\Delta M}$ tumor-bearing mice were more proliferative than WT B cells after treatment with LPS and IL-4 (Figure 5F). Malignant B cells from Becn $1^{4 M}$ tumor-bearing mice also displayed a unique phenotype, with increased expressions of B cell surface markers, including CD16/32 (FcYRIII/FcYRIIa), activation markers (CD80, CD86), and MHC class I (Figure 5G). Importantly, the elevated percentages of pre-B cells expressed low/intermediate B220 ( $16.8 \%$ in tumor vs. $0.78 \%$ in WT LN) and pre-B cell marker TdT (Figure 5, H and I). These pre-B cells were likely egressed from $\mathrm{BM}$ that displayed a loss of pre-B/immature B cells that migrated into secondary lymphoid organs (Figure 5, J and K, and Supple- mental Figure 3E). We found a small population of autoreactive $\mathrm{CD}^{+} \mathrm{B} 22 \mathrm{O}^{+}$double-positive cells proliferated in Becn $1^{4 M}$ mice, as an autoimmunity-prone phenotype observed in Fas-mutant lpr/ lpr lupus mice (ref. 22 and Figure $5 \mathrm{H}$ ). Consistently, Giemsa staining of BM cells showed a significant loss of lymphocytes, with dark nucleus staining and an increased number of granulocytes (Supplemental Figure 3F). Further, the cell-cell junction in bone sections of Becn1 $1^{1 M}$ mice was not as tight as in WT mice, as evident from H\&E staining (Supplemental Figure 3F). Since sphingosine-1-phosphate receptor (S1P1R) or CXCR4 controls B cell $\mathrm{BM}$ egression or retention $(23,24)$, respectively, we sorted B cells from both WT and Becn $1^{1 M}$ mice and found a marked reduction of CXCR4 expression, but not S1P1R, in B cells from Becn $1^{\Delta M}$ mice (Figure 5L), which might contribute to the pre-B cell BM egression.

Becn1-deficient neutrophils interact with B cells in tumor sites. By using immunohistochemical (IHC) staining and immunofluorescence (IF), we observed the colocalization of B cells with a large number of neutrophils in the tumor sites, lungs, and intestinal Peyer's patches (with a strip of neutrophils surrounding B cell zone) (Figure 6, A-C, and Supplemental Figure 4A). To determine the gene expression profiling (GEP) of the tumor and a potential role of neutrophils in tumor development, we extracted total RNA from cervical LN tumors for RNA-Seq and found many overexpressed genes in Becn $1^{4 M}$ mice, including those encoding inflammatory and B cell-stimulating molecules (Nos2, Ptgs2, Il21, Il1b, Il12, Tnfsf13b, Tnfsf18, Nlrp3), chemoattractants (Cxcl9, Cxcr3), neutrophil marker (Ly6g), and critical mediators of apoptotic cell removal (Supplemental Figure 4, B-E, and Supplemental Table 3). Transcriptional factors Ebf1 and Pax5, which have been reported to essentially contribute to the expansion of B cell progenitors and acute lymphoid leukemia transformation (25), were also upregulated (Supplemental Figure 4F). Notably, the downregulated genes included cell junction molecules related to leukocyte transendothelial migration (Cdh1, Cldn, Tjp1) (Supplemental Figure $4, \mathrm{~B}$ and $\mathrm{G})$, which might permit the noncanonical interactions between $B$ cells and activated myeloid cells.

Neutrophils have been illustrated as acquiring a B cell helper phenotype to promote B cell expansion and malignancy through several different mechanisms, including (a) contact-independent cytokine secretion, such as Tnfsf13b and IL-21, and (b) contactdependent mechanisms, such as CD40 and NETs (6, 7). Except for MCL-1 and BCL-2, marked upregulation of B cell helper signature genes was observed in Becn1-deficient neutrophils from tumor-bearing mice (Figure 6, D and E).

Furthermore, we found an increased accumulation of $\mathrm{Ly}^{6} \mathrm{G}^{+}$ neutrophils in the tumor (LN and lung infiltrates) with autoactivation of $\mathrm{p} 38$, which might contribute to IL-21 production (Figure $6 \mathrm{~F})$. Since myeloid-derived suppressor cells (MDSCs) expressed Gr-1 (Ly6C and/or Ly6G) and CD11b molecules are known to dampen immune response by inhibiting $\mathrm{CD}^{+}$and $\mathrm{CD}^{+} \mathrm{T}$ lymphocyte function in cancer, we tested to determine whether the accumulation of $\mathrm{Ly} 6 \mathrm{G}^{+} \mathrm{CD} 11 \mathrm{~b}^{+}$cells in Becn $1^{4 M}$ mice has a suppressive function. The $\mathrm{T}$ cell proliferation assay (determined by the incorporation of $\left[{ }^{3} \mathrm{H}\right]$ thymidine) showed that neutrophils $\left(\mathrm{Ly}_{6 \mathrm{G}}{ }^{+}\right)$ isolated from Becn1 ${ }^{\Delta M}$ mice (either with or without B cell lymphoma) had no significant suppressive effect on $\mathrm{T}$ cell proliferation (Supplemental Figure 4, $\mathrm{H}$ and I). 
A
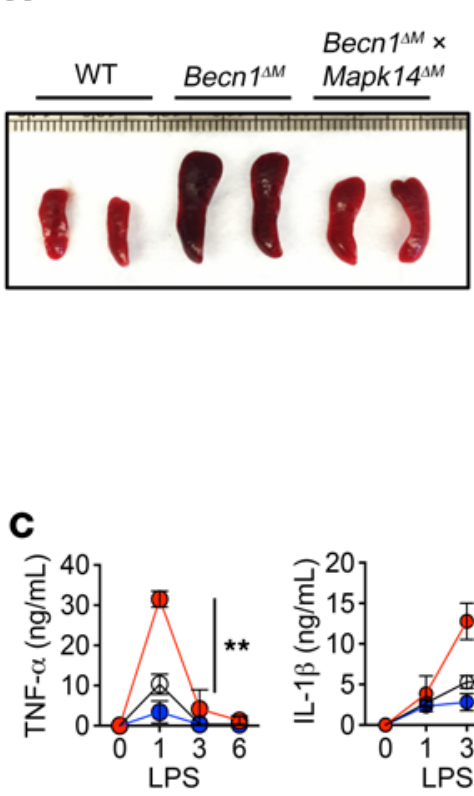

treatment $(\mathrm{h})$

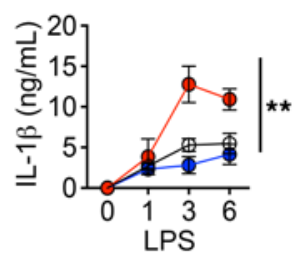

treatment (h)

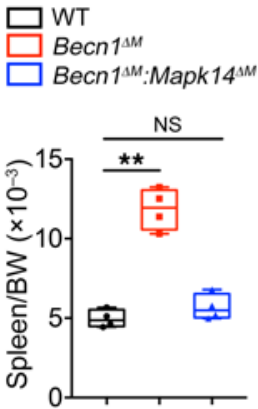

B

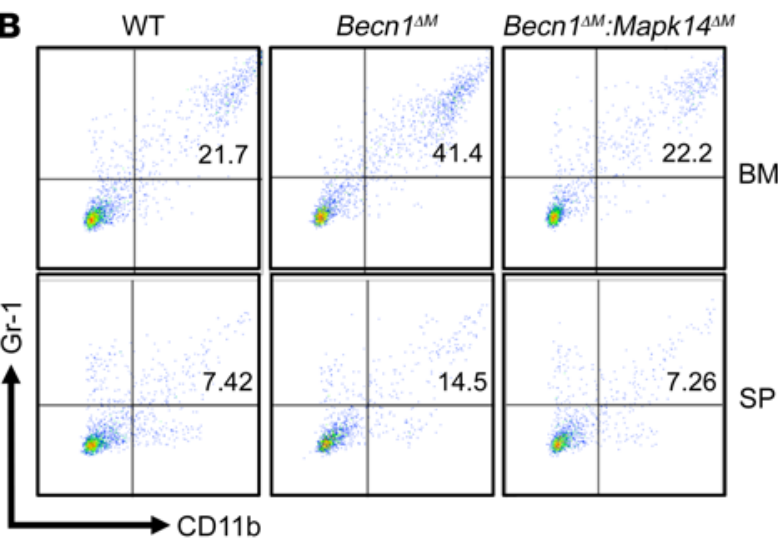

D
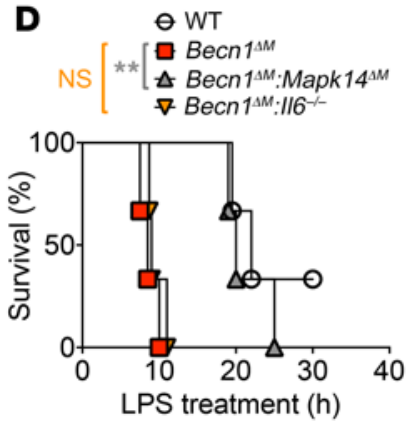

E

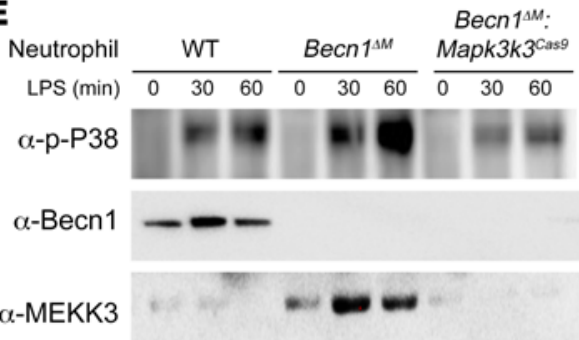

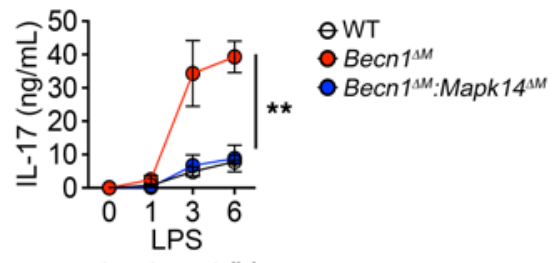

treatment $(h)$

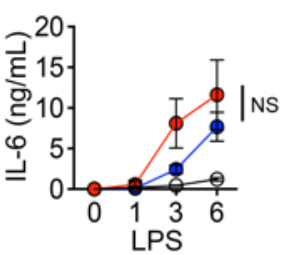

treatment (h)
$\mathbf{F}$

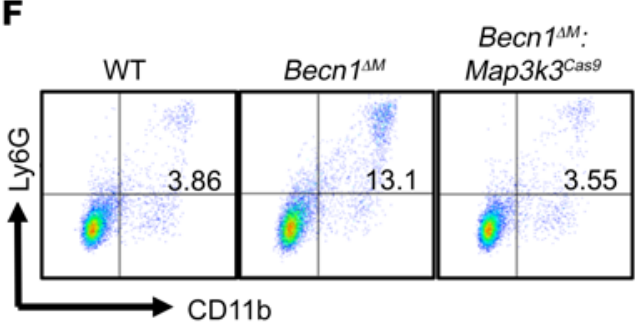

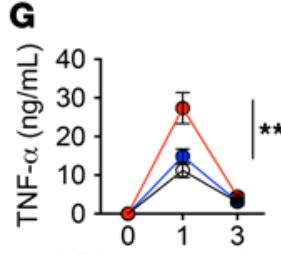

LPS treatment (h)

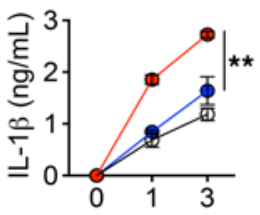

LPS treatment (h)

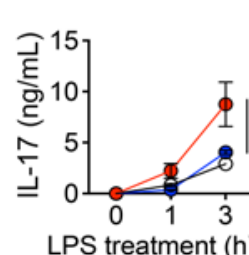

$\ominus$ WT

- Becn $1^{\text {sm }}$

- Becn $1^{\Delta M}: M a p 3 k 3^{\text {Cas } 9}$

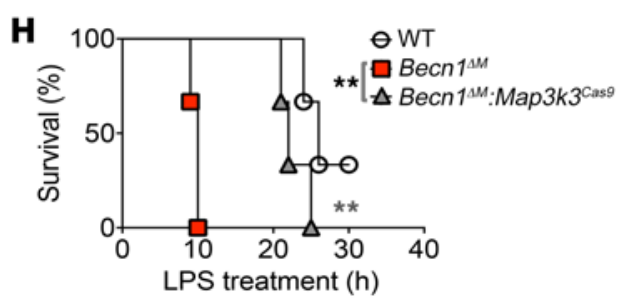

Figure 4. p38 or MEKKK3 ablation restores Becn1 ${ }^{1 M}$ mouse phenotype so that it is similar to WT. (A) SP size (left) and SP/body weight ratio (right) isolated from

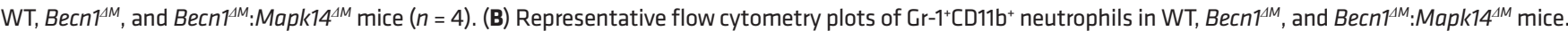

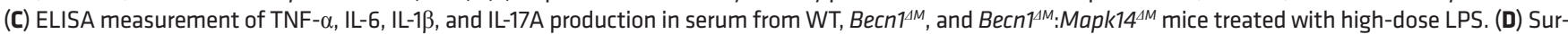

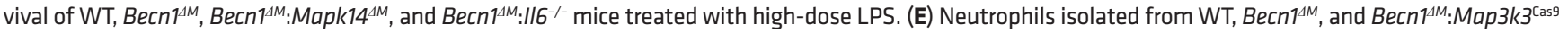
mice treated with LPS for the indicated time points, followed by IB with indicated antibody. (F) Representative flow cytometry plots of CD11b+Ly6C ${ }^{+}$neutrophils in WT, Becn $1^{1 M}$, and Becn1 ${ }^{1 M}: M a p 3 k 3^{\text {Cas9 }}$ mice. (C) ELISA analysis of TNF- $\alpha$, IL-1 1 , and IL-17A production in serum from WT, Becn ${ }^{1 M}$, and Becn $7^{1 M}: M a p 3 k 3^{\text {Cas9 }}$ mice treated with high-dose LPS. (H) Survival of WT, Becn ${ }^{1 M}$, and Becn $1^{1 M}: M a p 3 k 3^{\text {Cas9 }}$ mice treated with high-dose LPS. Data are representative of 3 independent experiments with 6- to 8-week-old mice ( $n=3$; female) (B-D) and 10- to 12-week-old mice ( $n=3$; female) (E-C). Statistical differences between groups were calculated using 1-way ANOVA with Dunnett's multiple comparison test (A, C, and $\mathbf{G})$ and Mantel-Cox log-rank test $(\mathbf{D}$ and $\mathbf{H}) .{ }^{*} P<0.05$; ${ }^{* *} P<0.01$.

Staining of Becn1-deficient neutrophils derived from tumorbearing mice with SYTOX impermeable DNA dye revealed an increased projection of NETs (Figure 6G). Thus, the recruitment of neutrophils by B cells through $\mathrm{Cxcl} / \mathrm{Cxcr3}$ chemotaxis (Figure $2 \mathrm{H}$ and Figure 6, $\mathrm{H}$ and I) might provide extensive survival signals and an inflammatory environment, leading to the malignant transformation of B cells.
Neutrophil with deficiency of Becn1 induces $P D-L 1$ in pre-B cell lymphomas. To determine how the myeloid-specific loss of Becn1 contributes to the malignancy of B cells, we isolated the total B cells from WT and Becn1 ${ }^{1 M}$ tumor-bearing mice for RNA-Seq and real-time PCR analysis. Marked upregulation of different genes was observed, including (a) STAT target genes, such as Cxcl9, Socs1, Socs3, Irf1, and Cd274 (PD-L1), (b) B (pre-B) cell markers, 

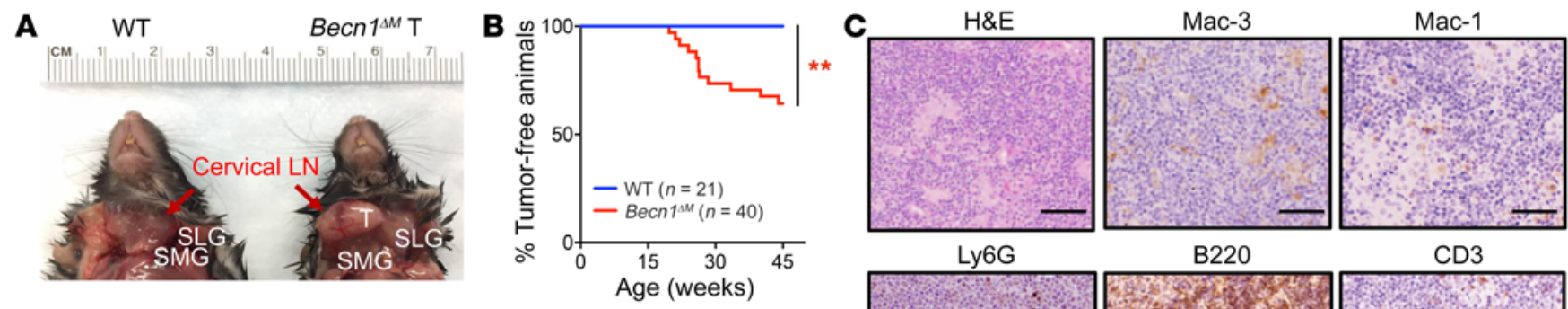

D
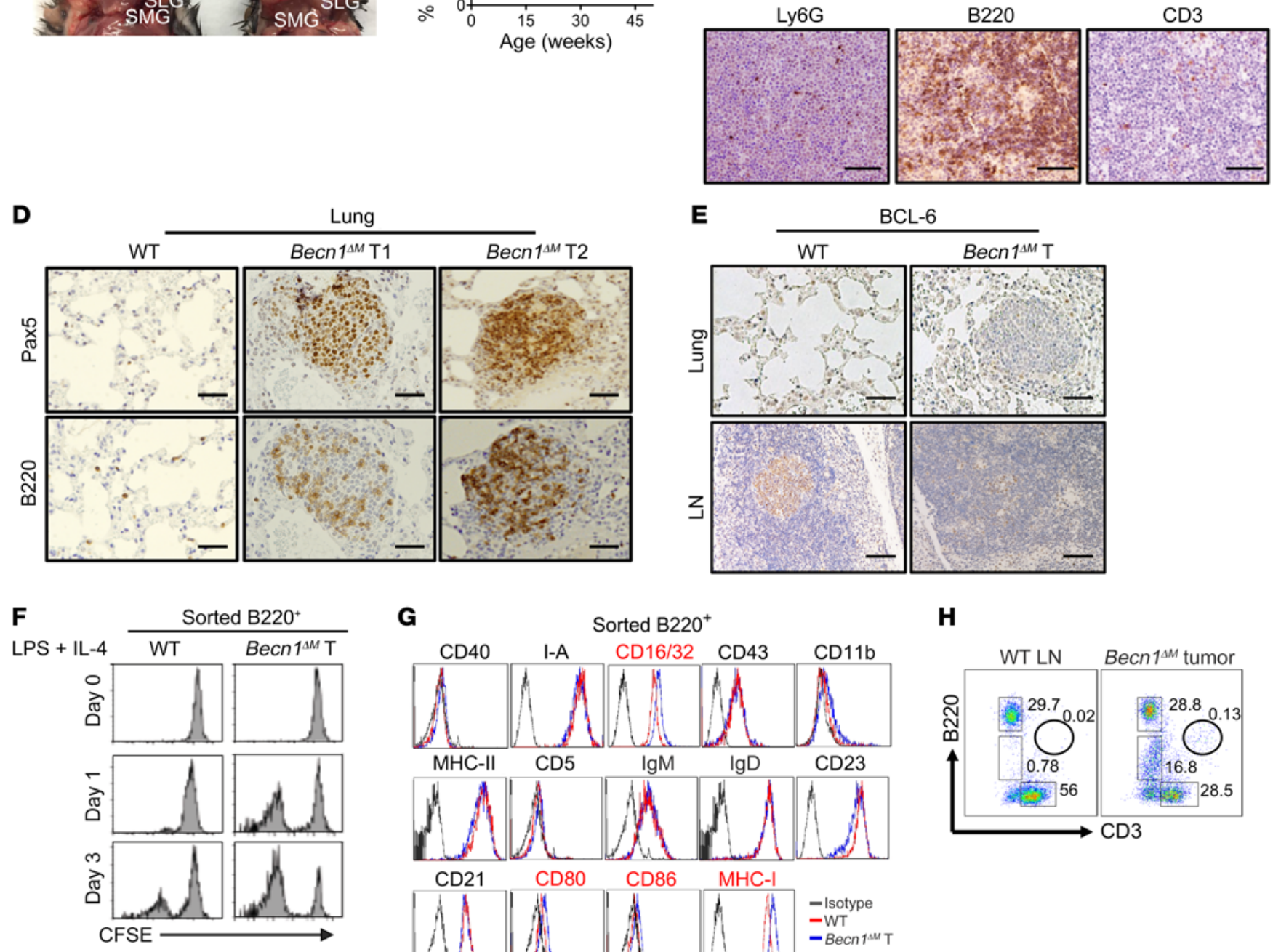

G

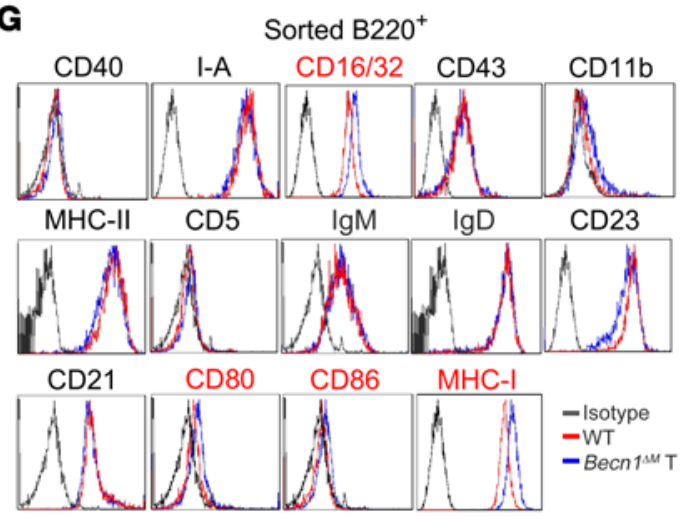

H
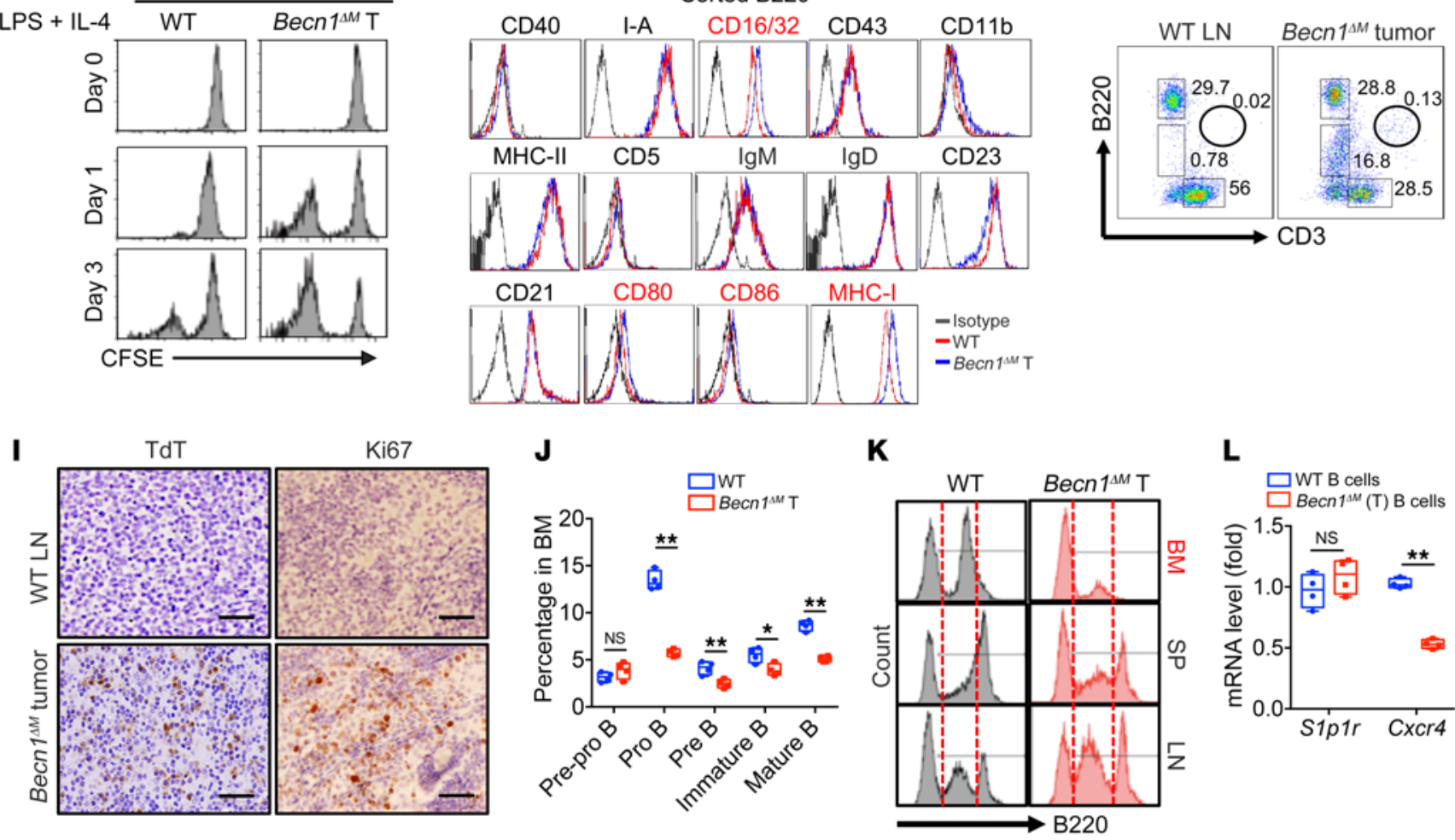

$\mathbf{L}$

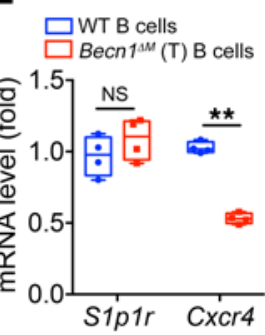


Figure 5. Identification of MHC-I $\mathrm{I}^{\mathrm{h}} \mathrm{B} 22 \mathrm{O}^{10} \mathrm{TdT}^{+}$pre-B cell lymphoma in Becn ${ }^{\mathrm{AM}}$ mice. (A) Tumor formation in cervical LN (red arrows) in Becn $1^{\mathrm{AM}}$ mice. T, tumor; SLG, sublingual gland; SMG, submandibular gland. (B) Kaplan-Meier curves for tumor-free animals were calculated based on the tumor latency of the controls ( $n=21 ; 11$ females, 10 males; blue) and $B e c n 1^{1 M}$ mice ( $n=40$; 31 females, 9 males; red). (C) IHC staining of tumor sections from Becn ${ }^{1 \mathrm{M}}$ tumor-bearing mice with indicated antibodies. Scale bars: $500 \mu \mathrm{m}$. (D) IHC staining of lung sections stained with B cell markers. $\mathrm{T} 1$, low antibody concentration; T2, high antibody concentration. Scale bars: $100 \mu \mathrm{m}$. (E) IHC staining of lung sections stained with anti-Bcl-6 (LN follicular B cells as a positive control). Scale bars: $100 \mu \mathrm{m}$. (F) FACS analysis of CFSE-labeled (APC-CFSE) B cells treated with LPS and IL-4 at indicated time points. (G) FACS analysis of B cell surface markers. Significant right-shift markers are indicated in red. (H) FACS analysis of cell populations in tumor with indicated antibody. (I) IHC staining of tumor sections with TdT and Ki67 antibodies. Scale bars: $100 \mu \mathrm{m}$. (J) FACS and statistical analysis of $B M$ pre-pro $B$ cell $\left(B 220^{+} C D 43^{+}\right)$, pro-B cell $\left(B 220^{+} C D 43^{10}\right)$, pre-B cell (B220 $\left.{ }^{+} \mathrm{CD}^{-} 3^{-}\right)$, immature $B$ cell $\left(B 220^{+} \mathrm{CD}^{-} 3^{-} \lg \mathrm{M}^{+} \lg \mathrm{D}^{-}\right)$, and mature $B$ cell (B220+CD43-lgM+lgD+) populations. (K) FACS analysis of B220+ $B$ cell population in $\mathrm{BM}, \mathrm{SP}$, and $\mathrm{LN}$ of $\mathrm{WT}$ and $B e c n 7^{1 \mathrm{M}}$ tumor-bearing mice. (L) Quantitative reverse-transcription PCR (qRT-PCR) of S1p1r and Cxcr4 mRNA in B220+ cells from WT $(n=5)$ and Becn $7^{1 M}$ tumor-bearing mice $(n=$ 9). Data are presented as box plots, with error bars showing mean \pm SEM (J and $\mathbf{L}$ ) and are representative of 3 independent experiments ( $n=3$; female) (A, C-I, and K). Statistical differences between groups were calculated using Mantel-Cox log-rank test (B) and Student's unpaired $t$ test ( $\mathbf{J}$ and $\mathbf{L}$ ). ${ }^{*} P<0.05 ;{ }^{* *} P<0.01$.

such as IL2ra and Ly6a (26), (c) B cell activation markers, such as IL10 and Saa3, (d) MHC class I-related molecules, such as Nlrc5 and Psmb9, and (e) innate immunity gene Mb21d1 (cGAS), known to suppress homologous recombination-mediated DNA repair in nucleus and promote tumorigenesis (ref. 27, Figure 7, A and B, and Supplemental Table 4).

We next sought to determine whether the tumor cells had altered oncogenic signaling, as suggested in previous studies $(28,29)$. The tumor and control cells were sorted by B220-positive selection, followed by immediate lysis or anti-CD40 agonist stimulation. We observed elevated activations of ERK and JNK; downstream signaling of IL-21 (p-STAT1, p-STAT3, p-STAT5, with total STAT3 unchanged); and BAFF (noncanonical NF-кB p52); as well as upregulated STAT5-target (antiapoptotic gene MCL-1) (ref. 30 and Figure 7C and Supplemental Figure 5A). A recent study demonstrates that hyperactivation of AKT by PTEN ablation triggers the death of pre-B cells (26). However, we did not observe the changes of AKT and FOXO1 phosphorylation in these B cells (Supplemental Figure 5A). Consistent with the upregulation of MCL-1, we observed reduced apoptosis of B cells isolated from the tumor, but not the non-tumor bearing Becn $1^{\Delta M}$ mice (Supplemental Figure $1 \mathrm{G}$ and Supplemental Figure 5B). Moreover, we compared the signaling activations in $\mathrm{B}$ cells from WT, tumor-free Becn $1^{\Delta M}(\mathrm{NT})$, and $\mathrm{B} 22 \mathrm{O}^{\text {hi }}$ versus $\mathrm{B} 22 \mathrm{O}^{\text {lo }}$ cells from tumor-bearing Becn $1^{\Delta M}$ mice and found that $\mathrm{B} 22 \mathrm{O}^{\mathrm{lo}}$ cells had higher levels of phosphorylated STAT1/3/5 and PD-L1 than B220 ${ }^{\text {hi }}$ cells (Figure 7, D and E). By coculturing the neutrophils or macrophages from tumor-bearing $B e c n 1^{4 M}$ mice with normal WT B cells, we found the induction of PD-L1, as well as CXCR3 ligands Cxc19/10, in B cells by neutrophil-derived factors (Supplemental Figure 5, C and D). Further, the upregulation of PD-L1 could be abolished by antibody blockade of either IL-21 or CD4OL (Supplemental Figure 5E). To under- stand the signaling pathways in B cells that control the expression of PD-L1, we applied MAPK inhibitors (p38i, SB 203580; ERKi, U0126; JNKi, sp 600125), NF-אB inhibitor (BAY 11-7082), and STAT3 inhibitor (STAT3i, WP 1066) to sorted B cells after coculture with Becn1-deficient neutrophils. The inhibition of ERK and STAT3 was able to reduce the PD-L1 level, suggesting that both ERK and STAT3 signaling pathways are involved in the regulation of PD-L1 expression (Supplemental Figure 5F). We also detected the upregulation of IRF1 in B cells (Figure 7, A and B). IRF1 is an essential transcription factor that regulates PD-L1 and MHC-I expression $(31,32)$. In addition, ERK signaling promotes PD-L1 expression through the negative regulation of tristetraprolin (TTP, encoded by Zfp36) expression and activity, which is a zinc-finger RNA-binding protein that suppresses PD-L1 mRNA through binding to the AU-rich element at 3' UTR (33). By inhibition of STAT3 and ERK signaling, we observed reduced levels of Irf1 (by STAT3i) and increased levels of Zfp36 (by ERKi) (Supplemental Figure $5 \mathrm{G}$ ), suggesting that the activations of both ERK/TTP and STAT3/ IRF1 signaling by neutrophil-derived factors contributed to the upregulation of PD-L1 expression in pre-B cell lymphoma cells. Notably, no change was observed in the expression of Cmtm4/6, which stabilize the PD-L1 protein (34), in B cells after coculture with Becn1-deficient neutrophils (Supplemental Figure 5H).

To further demonstrate the effects of neutrophil factors that promote PD-L1 expression in vivo, we treated Becn $1^{4 M}$ mice (6 to 8 weeks old without tumors) by i.p. injection of recombinant neutrophil factors (Supplemental Figure 6A) and found that IL-21 significantly promoted the enlargement of cervical LN as well as PD-L1 expression in sorted $\mathrm{CD} 19^{+}$or $\mathrm{B} 22 \mathrm{O}^{+} \mathrm{B}$ cells (Supplemental Figure 6B). In contrast, irradiated Becn1 $1^{\Delta M}$ mice reconstituted with $\mathrm{BM}$ from tumor-bearing Becn1 $1^{4 M}$ mice with IL-21 ablation (Becn1 $1^{4 M: I l 21}$ $\mathrm{KO})$ showed a reduction of PD-L1 expression and STAT3 activation (Figure 7, F and G). To further examine the physiological role of PD-L1 molecules in cancerous pre-B cells in the regulation of the endogenous antilymphoma $\mathrm{CD}^{+} \mathrm{T}$ cells, we injected Becn1 $1^{\Delta M}$ tumor-bearing mice with antibodies against PD-L1 or IL-21R, compared them with mice injected with IgG control, and found a significantly increased number of total $\mathrm{CD} 8^{+}$cells as well as $\mathrm{CD}^{+} \mathrm{IFN}^{-} \gamma^{+}$and $\mathrm{CD}^{+} \mathrm{Granzyme}^{+} \mathrm{T}$ cells in the tumor region (Supplemental Figure 6, C and D).

Since p38 signaling regulated neutrophil inflammation in $B e c n 1^{\Delta M}$ mice in response to TLR ligand stimulation (Figures 2 and 4), which was autoactivated in tumor-derived neutrophils (Figure $6 \mathrm{~F}$ ), we next determined whether ablation of p38 could reduce the expressions of neutrophil-derived $\mathrm{B}$ cell activation factors (e.g., IL-12, CD40L, IL-1 $\beta$, IL-21, and BAFF), immunosuppressive molecule PD-L1, and the risk of B cell lymphoma in Becn $1^{1 M}$ mice. Indeed, myeloid-specific ablation of p38 in Becn $1^{\Delta M}$ mice restored the expression of neutrophil-derived $\mathrm{B}$ cell activation factors, reduced STAT3 phosphorylation and PD-L1 expression and B cell $\mathrm{BM}$ egression, and in turn, suppressed spontaneous tumor development in Becn1 $1^{\Delta M}$ mice (Supplemental Figure 6, E-H).

To investigate whether Becn1 in human neutrophils plays a role in human B cell lymphoid malignancies, we first analyzed Becn1 expression in a series of human B cell non-Hodgkin lymphomas (B-NHL) and pre-B cell ALL (without mutations or gene rearrangements) by human GEP. Becn1 expression levels varied 


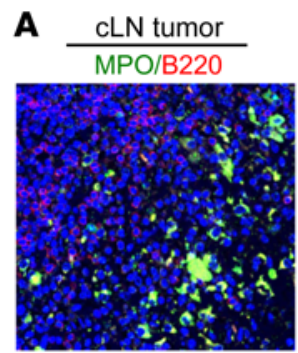

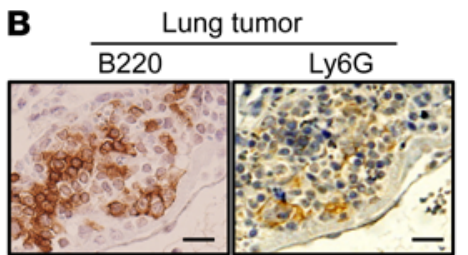

MPO/B220/DAPI

1
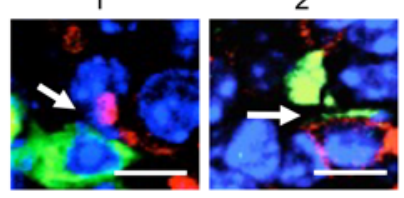

C
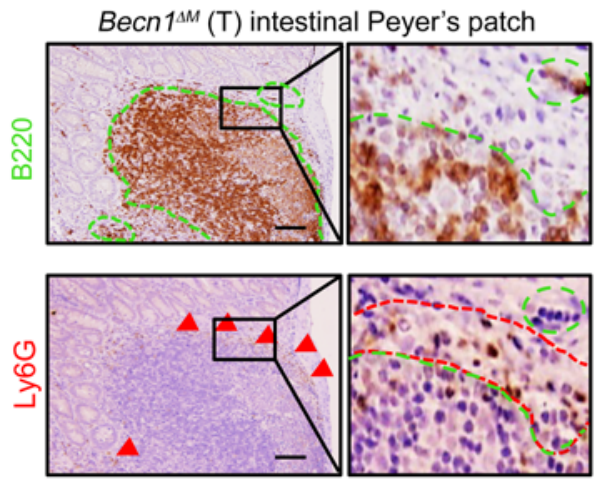

Proliferation and migration

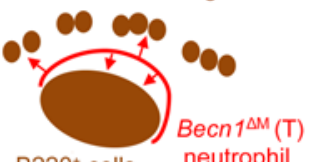

D

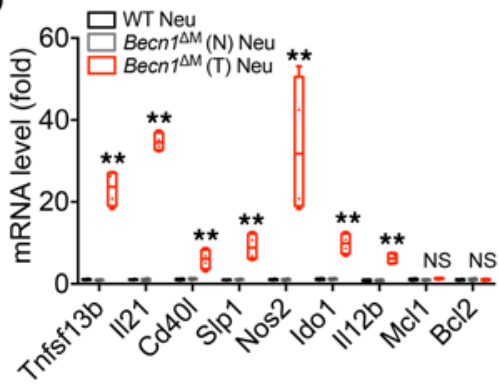

\section{E $\square$ WT Neu}

$\square$ WT Neu
$\square{\text { Becn } 1^{\Delta M} \text { (N) Neu }}^{\Delta \text { Becn }^{\Delta M} \text { (T) Neu }}$

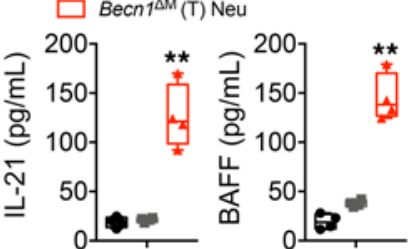

a

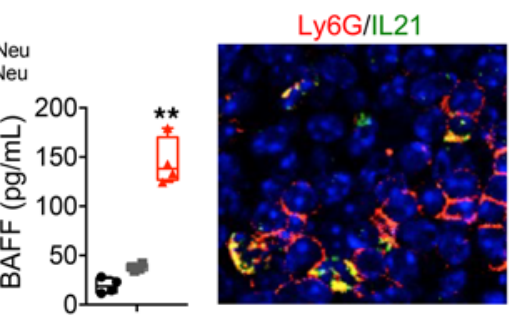

NET

F
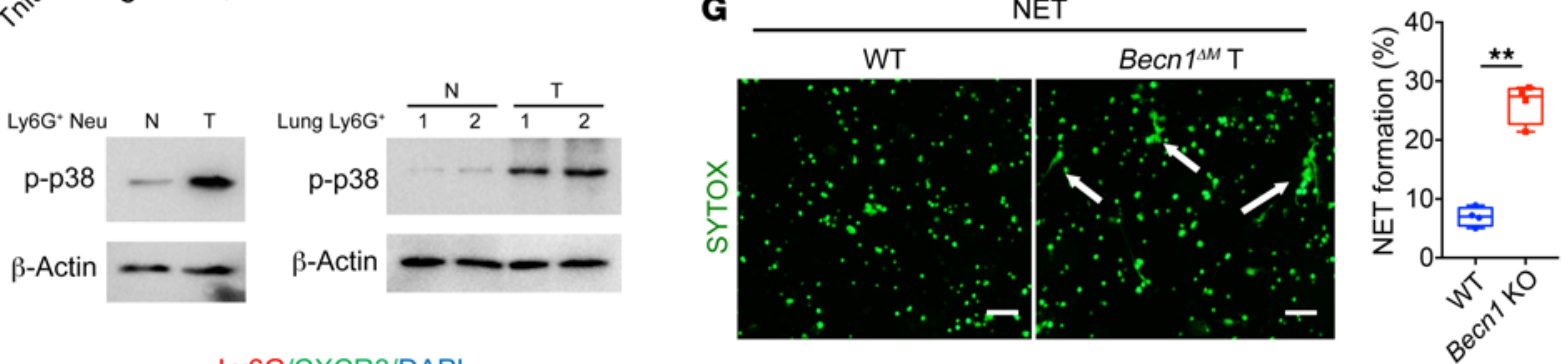

H

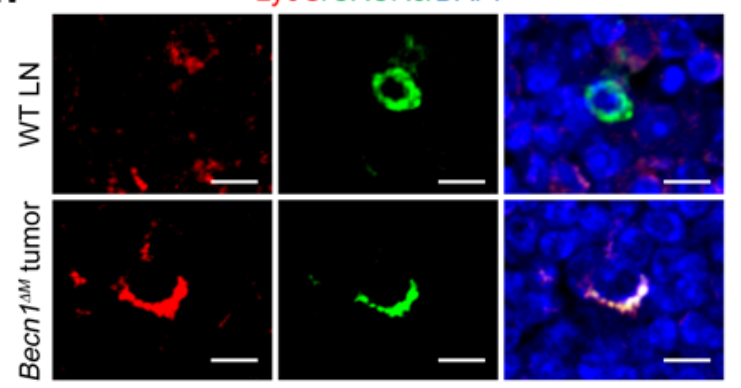

I

CXCL9

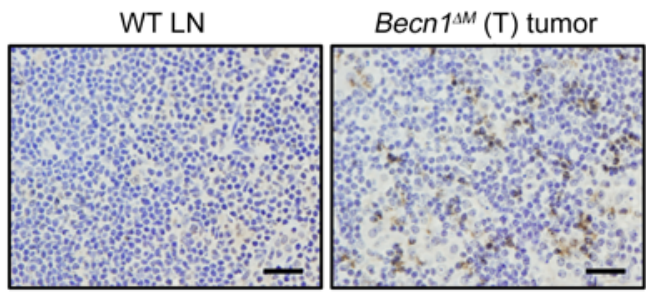

Figure 6. Becn1 deficiency induces B cell helper protumorigenic function of neutrophils. (A and B) IHC staining or IF of tumor in cLN and lung stained with indicated antibody showing the interaction of B cells with neutrophils (arrows). DAPI, DNA-intercalating dye (nucleus, blue). Scale bars: $25 \mu \mathrm{m}$. (C) IHC staining of intestinal Peyer's patch sections with indicated antibodies (green circle indicates B cell zone; arrowheads indicate neutrophils). Scale bars: 500 $\mu \mathrm{m}$. Drawing illustrates a strip of neutrophils surrounding the edge of the B cell zone likely to support B cell proliferation and migration. (D) qRT-PCR of B cell helper neutrophil signature genes. (E) ELISA analysis of BAFF and IL-21 production by neutrophils after in vitro culture for 12 hours. IF shows accumulation of $\mathrm{Gr}-1^{+} \mathrm{CD}_{11 \mathrm{~b}^{+}}$neutrophils in the tumor expressing IL-21. Original magnification, $\times 20$. (F) IB of p38 activation in neutrophils from lymphoma (T) and lungs in Becn $1^{1 \mathrm{M}}$ tumor-bearing mice compared with WT controls. N, normal. (C) Fluorescence and statistical analysis of NET formation in neutrophils using SYTOX dye (green). White arrows indicate NETs. Scale bars: $100 \mu \mathrm{m}$. (H) IF of tumor section with Ly6G (red) and CXCR3 (green) antibodies compared with WT LN. Scale bars: $25 \mu \mathrm{m}$. (I) IHC staining of tumor sections with anti-CXCL9 antibody compared with WT LN. Scale bars: $200 \mu \mathrm{m}$. Data are presented as box plots with error bars showing mean \pm SEM (D, E, and $\mathbf{G})$ and are representative of 3 independent experiments $(n=3$; female) $(\mathbf{A}-\mathbf{C}, \mathbf{F}, \mathbf{H}$, and $\mathbf{I})$. Statistical differences between groups were calculated using 1-way ANOVA with Dunnett's multiple comparison test (D and $\mathbf{E})$ and Student's unpaired $t$ test $(\mathbf{G}) .{ }^{*} P<0.05$; ${ }^{* *} P<0.01$.

significantly among the different B cell lymphoid malignancies, with the lowest levels in pre-B cell ALL and the highest in mantle cell lymphoma (MCL) and chronic lymphocytic leukemia (CLL) (Supplemental Figure 6I and Supplemental Table 5). Furthermore, we found lower Becn1 expression in neutrophils among human pre-B cell ALL patients compared with normal controls (Figure $7 \mathrm{H})$. Notably, in recurrent pre-B cell ALL patients, neutrophils with low Becn1 expression had a NET-like structure (Figure 7H). 
Consistent with the upregulation of PD-L1 in B cells from Becn1 ${ }^{4 M}$ mice, we found upregulation of PD-L1 in pre-B cell ALL patient $\mathrm{BM}$ and LN samples, especially in recurrent pre-B cell ALL samples (Figure 7I). In a clinical study of B-cell ALL patients (Therapeutically Applicable Research to Generate Effective Treatment [TARGET] ALL projects), significant positive correlations were observed in the expression of IL-21, PD-L1 (CD274), and neutrophil markers (human ortholog LY6G6D and CD177) (refs. 35, 36, Figure 7J, and Supplemental Figure 6J). These data suggest that low Becn1 expression in neutrophils might have a direct link to PD-L1 level expression and recurrence in human pre-B cell ALL. Thus, our results suggest that the low Becn1 expression in neutrophils correlates with PD-L1 levels in pre-B cell ALL patients.

\section{Discussion}

Despite the importance of autophagy in macrophages during Mycobacterium tuberculosis infection or LC3-associated phagocytosis $(37,38)$, little is known about the in vivo function of Becn1 in neutrophils regarding inflammation and cancer immunity. Our study showed that deletion of Becn1 in the myeloid lineage led to neutrophilia, which caused splenomegaly and lymphadenopathy in mice. We uncovered a cell type-specific mechanism of Becn1 as a negative regulator of p38 signaling in neutrophils. Depletion of neutrophils, but not macrophages, could rescue the sensitivity of $B e c n 1^{\Delta M}$ mice to LPS endotoxin shock, suggesting excessive LPSinduced neutrophil inflammation. Our cytokine profiling and RNA-Seq analyses further revealed the enhanced production of TNF- $\alpha$, IL-1 $\beta$, ROS, IL-17-related cytokines, and chemokine-receptor CXCR3 by neutrophils in Becn1 $1^{M M}$ mice. p38 MAPK signaling is critical for the transcription and production of inflammatory cytokines $(39,40)$. Consistent with the cell-depletion results, we provided compelling genetic evidence that p38 (encoded by Mapk14) ablation in myeloid cells rescued the observed phenotypes and the production of inflammatory cytokines in Becn1 $1^{1 M}$ mice. NF- $\mathrm{KB}$ activation is known to negatively regulate IL-1 $\beta$ secretion in neutrophils in myeloid IKK $\beta$-deficient mice (41). Consistently, we observed reduced IKK phosphorylation and increased IL-1 $\beta$ secretion upon LPS stimulation in Becn1-deficient neutrophils. Despite the elevated production of IL- 6 in macrophages, IL- 6 ablation failed to rescue the survival of Becn1 ${ }^{4 M}$ mice from LPS-induced septic shock.

It is known that MAP3K (TAK1, ASK1, MEKK3) activation triggers downstream signaling of IKK complex, Erk1/2, p38, and JNK (18). We showed that Becn1 interacted with MEKK3 and MKK3 and promoted the MEKK3 K48 ubiquitination at K299 and subsequent autophagy-independent proteasomal degradation. Due to the low protein level of MEKK3 observed in neutrophils, the activation of p38 in neutrophils is mainly triggered by upstream molecules other than MEKK3, such as TAK1. The stabilization of MEKK3 upon Becn1 deletion thus leads to aberrant activation of neutrophils through the MEKK3/MKK3/p38 signaling axis. Consistently, further ablation of MEKK3 significantly prolonged the survival of Becn $1^{4 M}$ mice from LPS-induced septic shock.

Heterozygous deletion of Becn1 increases the incidence of spontaneous tumors in mice, including diffused large cell B cell lymphoma (DLBCL) (3), suggesting that Becn1 is a haploinsufficient tumor suppressor. However, it is unclear whether the tumor formation is induced by intrinsic factors or by oncogenic inflam- mation from nontransformed immune cells in the tumor microenvironment. While Becn1 expression is intact in pre-B cells, Becn1 $1^{\Delta M}$ mice showed a high risk of $\mathrm{TdT}^{+}$pre-B cell lymphoma formation ( $40 \%$ frequency) as early as the age of $15-25$ weeks. The accumulation of neutrophils, but not macrophages, was found to colocalize and interact with malignant B cells in tumor sites. It is worth noting that neutrophilia developed in mice with or without lymphoma, probably due to $\mathrm{p} 38$ activation and high levels of IL-1 $\beta$, which could cause the accumulation of neutrophils through IL-1R1 signaling (42). Although Becn1 deficiency in myeloid cells did not alter the number and overall infiltration of macrophages, these macrophages are prone to display M1 phenotypes known to be antitumor (43). Furthermore, we found that the pre-B tumor cells were egressed from BM. It has been reported that stromal cells, Bruton's tyrosine kinase, the S1P/S1P receptor, CXCL12/CXCR4 signaling cascades, and $\alpha 4 \beta 1$-VCAM-1-mediated adhesion could all regulate the $\mathrm{B}$ cell migration from $\mathrm{BM}(7,44-47)$. Our data suggest that the $\mathrm{BM}$ (a major pool of myeloid cells, such as neutrophils) is a critical location for pre-B cells to be activated by local inflammatory neutrophils in tumor-bearing Becn $1^{4 M}$ mice. This unique interaction led to the egression of pre- $\mathrm{B}\left(\mathrm{B} 22 \mathrm{O}^{\mathrm{lo}}\right.$ ) cells from BM (by downregulation of BM retention signal Cxcr4, but not the S1P egression signal), serving as a prerequisite step for the development of metastatic pre-B cell lymphoma in cervical LNs and lungs. We reason that the IL-21 produced by tumor-associated neutrophils and subsequent STAT3/SOCS3 activation in B cells are the potential mechanisms to control the lodgment of pre-B cells in the BM, as it could downregulate CXCR4 signaling $(48,49)$.

Recent studies also identify a unique population of IL-21secreting neutrophils, termed B cell helper neutrophils, to sustain B cell survival, expansion, and malignant transformation (7, 8). IL-21 receptor signaling has been linked to inflammation and autoimmune diseases, such as SLE (50). IL-21 also enhances inflammation-associated malignancy (51). However, the potential mechanisms underlying the differentiation and transition of neutrophils to gain "B cell helper" function remain unknown. In this study, we showed that Becn1 is a key regulator governing the transition of normal neutrophils into protumoric neutrophils with upregulated B helper signatures, including IL-21, BAFF, CD4OL, and the production of NET. These factors have been shown to activate multiple oncogenic signaling pathways, such as STATs and noncanonical NF- $\mathrm{BB}$, that are crucial in initiating oncogene transcription and establishing the tumor premetastatic niche (29, 52-54). Therefore, myeloid deficiency of Becn1 provides a unique model for investigating the neutrophil/B cell interaction.

Strikingly, when comparing B220 ${ }^{\mathrm{lo}}$ with $\mathrm{B} 220^{\text {hi }}$ cell populations, we showed that $\mathrm{B} 22 \mathrm{O}^{\mathrm{lo}}$ cells had higher and persistently activated STAT1, STAT3, STAT5, and noncanonical NF-кB p52, followed by the upregulation of their downstream targets MCL-1, chemokine Cxcl9, and checkpoint inhibitor PD-L1. The interaction with Becn1-deficient neutrophils also activated B220 ${ }^{\text {hi }}$ cells in tumor sites, but to a lesser extent, compared with $\mathrm{B} 22 \mathrm{O}^{\mathrm{lo}}$ cells, suggesting the heterogeneity of these tumor cells. We also demonstrated that CD4OL/ERK and IL-21/STAT3-IRF1 were key signaling pathways for governing the expression of PD-L1 in pre-B cells. IL-21 treatment in Becn $1^{\triangle M}$ mice promoted lymphoma formation in cervical LN and PD-L1 expression on B cells. These findings are 

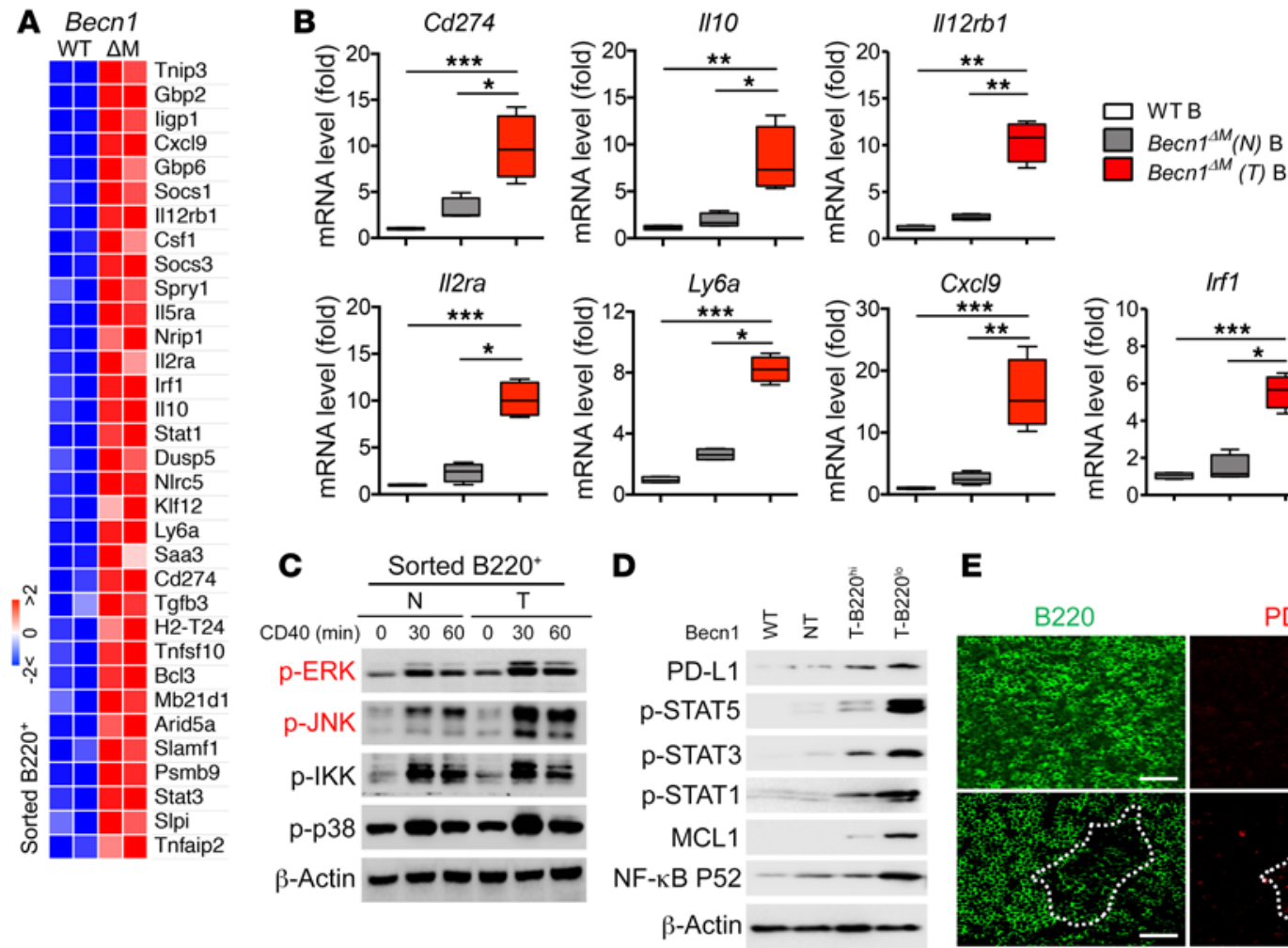

E
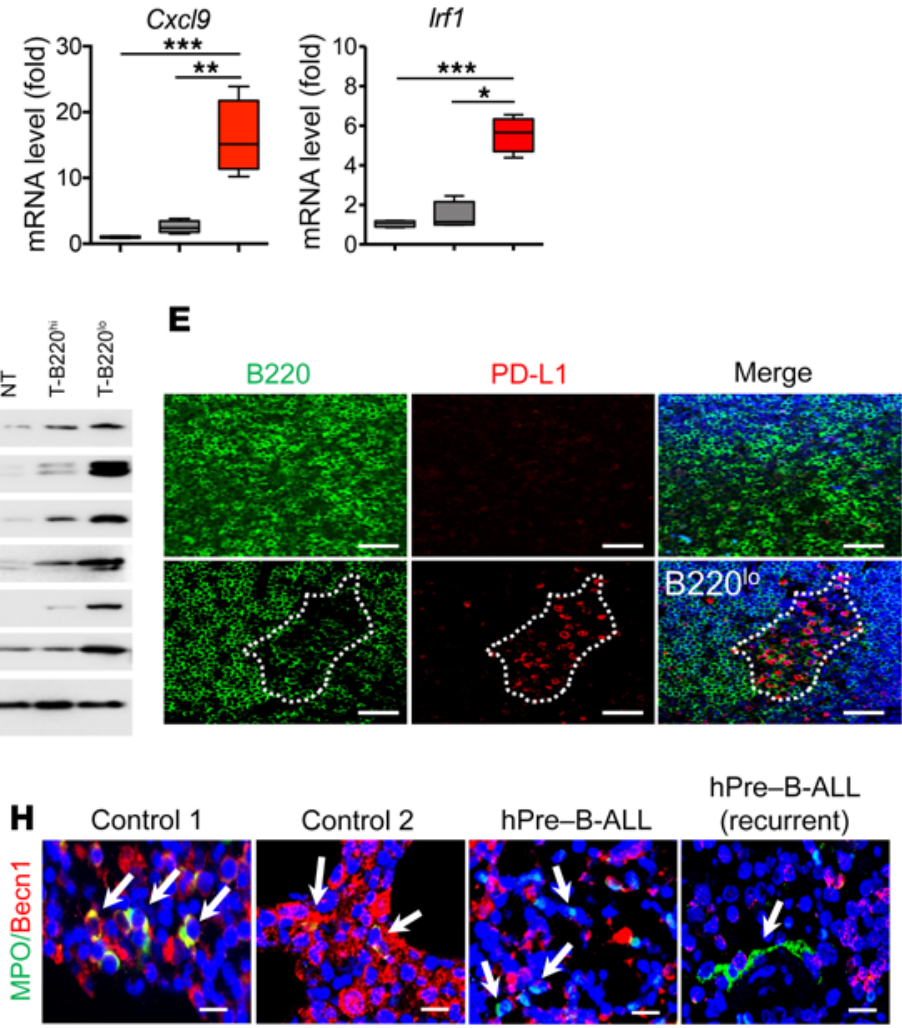

hPre-B-ALL
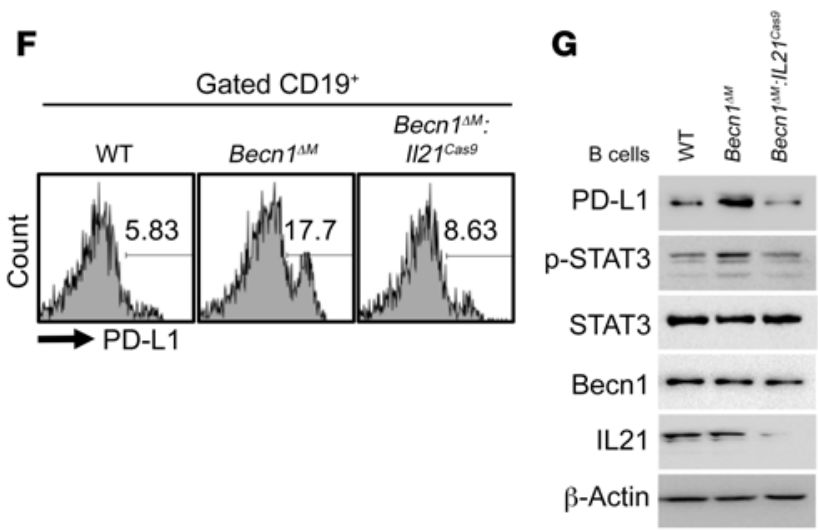

$\mathbf{F}$

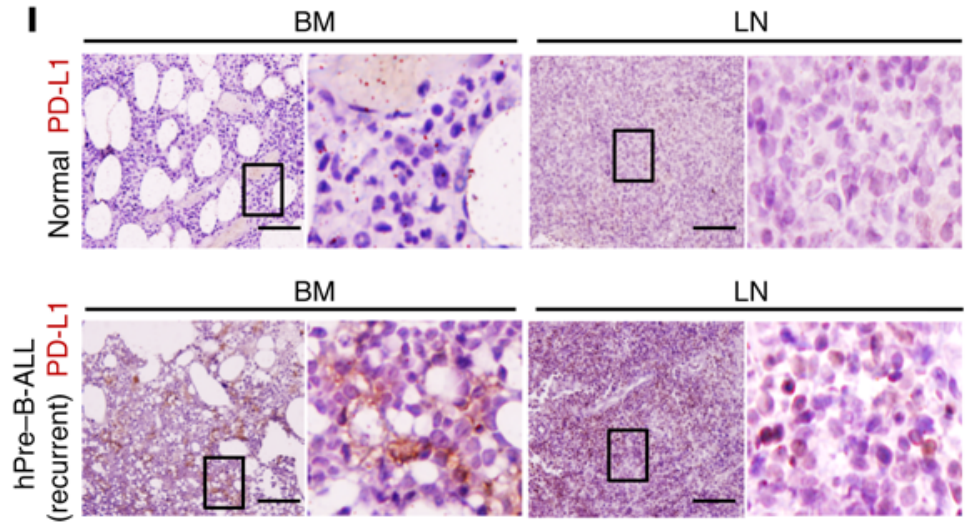

J ALL phase I (B-ALL)

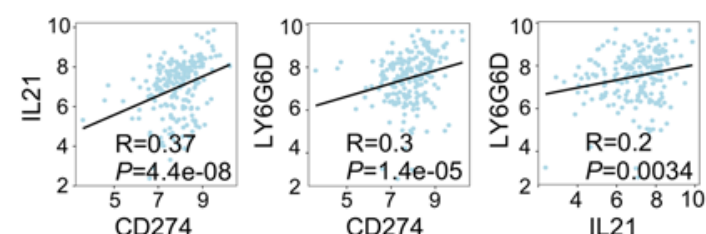

ALL phase II (B-ALL)

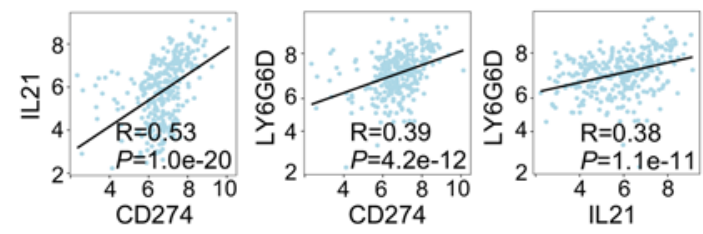


Figure 7. Neutrophil Becn1 levels correlate with PD-L1 in pre-B lymphoma. (A and B) Heatmap of upregulated genes in B cells with selected genes confirmed by qRT-PCR $(n=4)$. (C) IB of B220+ cells with indicated antibodies after stimulation with CD40 agonist. (D) IB of B cells with indicated antibodies comparing B220 ${ }^{\mathrm{hi}}$ and $\mathrm{B} 22 \mathrm{O}^{\mathrm{lo}}$ cells in tumor. (E) IF of tumor sections with anti-PD-L1 (red) and anti-B220 (green). Scale bars: $100 \mu \mathrm{m}$. (F) FACS analysis of PD-L1 expression in B cells from WT, Becn $1^{1 \mathrm{M}}$

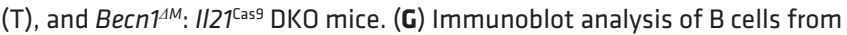
WT, Becn $1^{1 \mathrm{M}}$, and Becn $1^{1 \mathrm{M}}: \| 1 / 7^{\text {Cas9 }}$ DKO mice with indicated antibodies. (H) IF and statistical analyses of Becn1 expression in MPO+ cells with or without NET formation in pre-B-cell ALL compared with normal controls. Scale bars: $25 \mu \mathrm{m}$ (normal, $n=6$; pre-B-cell ALL, $n=10$ ). Arrows indicate MPO+ neutrophils. (I) IHC of PD-L1 expression in pre-B cell ALL samples $(n=10)$ compared with normal controls $(n=6)$. Scale bars: $100 \mu \mathrm{m}$. LN, reactive lymphoid hyperplasia. (J) Correlation of IL-21, PD-L1, and neutrophil marker (LY6G6D) in B-cell ALL data set (TARGET). Data are presented as box plots with error bars showing mean \pm SEM (B and $\mathbf{H}$ ) and are representative of 3 independent experiments ( $n=3$; female) (C-C). Statistical analysis using 1-way ANOVA with Dunnett's multiple comparison test (B) and Student's unpaired $t$ test $(\mathbf{H}) .{ }^{*} P<0.05 ;{ }^{* *} P<0.01 ;{ }^{* * *} P<0.001$.

consistent with the previous report that cytokine IL-21 induces PD-L1 expression in CD19+ B cells, but not in purified T cells (55). Based on these findings, we propose the feed-forward recruitment of neutrophils by pre-B cells via the CXCL9-CXCR3 chemotaxis to promote the development of immunosuppressive pre-B cell lymphoma. Notably, cGAS acts as a tumor enhancer in the presence of genotoxic or ROS stress through inhibiting homologous recombination-mediated DNA repair. Aberrant upregulation of cGAS transcripts has been reported in non-small cell lung carcinomas (27). Similarly, we showed that cGAS mRNA levels were upregulated in pre-B tumor cells. The potential association between the upregulation of cGAS in pre-B tumor cells and the malignant transformation of pre-B cells awaits further investigation.

PD-L1 is one of the critical checkpoint inhibitory signaling molecules utilized by tumor cells or other immune suppressor cells to inhibit $\mathrm{T}$ cell function for immune evasion (56). PD-L1-expressing $\operatorname{IgA}^{+} \mathrm{B}$ cells have been shown to suppress $\mathrm{CD} 8^{+} \mathrm{T}$ cells to kill liver tumor in a mouse model of inflammation-induced liver cancer (57). Administration of PD-L1 or IL-21R antibody in Becn1 $1^{1 M}$ tumor-bearing mice reversed the suppressive function of immunosuppressive PD-L $11^{\text {hi }}$ pre-B cells against $\mathrm{CD} 8^{+} \mathrm{T}$ cells. Injection of IL-21 increased the risk of developing lymphoma with upregulated PD-L1 in Becn $1^{1 \mathrm{M}}$ mice, suggesting the critical role of IL-21 in tumor immunity. Importantly, low Becn1 expression in tumor-infiltrated neutrophils correlates with PD-L1 levels in recurrent pre-B ALL patients. In addition, positive correlations between any 2 of the following 3 factors were observed in pre-B ALL patients: neutrophil marker Ly6g, IL-21, and PD-L1, which recapitulates the malignant pre-B cells identified in Becn $1^{M M}$ mice. Together, these findings indicate the potential of immunotherapy for the treatment of metastatic and/or recurrent pre-B ALL that acts by combining PD-L1 or IL-21 antibody blockade with inhibitors targeting tumor-infiltrating neutrophils. We also offer evidence to support the critical role of neutrophil-mediated inflammation in B cell migration and lymphomagenesis. Overall, our results provide molecular insights into the mechanisms of how Becn1-deficient neutrophils produce proinflammatory cytokines through regulation of p38 and MEKK3 signaling pathways and promote tumor development by inducing PD-L1 expression in pre-B cells. Thus, myeloid Becn1 may serve as a therapeutic target for cancer immunotherapy.

\section{Methods}

Animals and in vivo procedures. Adult C57BL/6 (CD45.2) and Boy/J (CD45.1) mice were obtained from the Jackson Laboratory. Becn $1^{f l}$ mice (Knockout Mouse Project [KOMP] Repository) were crossed with lysozyme-Cre (Lyz2-Cre) mice (Jackson Laboratory) to obtain $B e c n 1^{\Delta M}$ mice on the C57BL/6 background. Mouse genotyping primers to detect WT and flox/flox mutant were as follows: Becn1-1R: 5'-GCGGATCCCTGAGTTCTAGACTAACC-3'; Becn1-1F (ttR): CTCCCAATGCTGGGATTAAAGACG-3'; and Becn1-2R (F): TTGTACCGTGATTTAGGGCGTTTGC-3'. Becn $1^{4 M}$ mice were bred with Mapk14 ${ }^{l}$ mice (provided by Y. Wang, UCLA, Los Angeles, California, USA, and $\mathrm{H}$. Jiang, Boehringer-Ingelheim) or $I l 6^{-/-}$mice (Jackson Laboratory) to generate Becn1 $1^{\Delta M}: M a p k 14^{\Delta M}$ and Becn1 $1^{\Delta M}: I l 6^{-/-}$mice, respectively. To generate Becn1 and Map3k3, Becn1 and Il21 DKO BM chimeric mice, deletions of Map3k3 and Il21 were achieved by a CRISPR-Cas9 system (20). We used the following sgRNA sequences to target exon1 of Map3k3: ACCGGGTTCGTCGGCTCATC and exon1 of Il21: Il21sg-RNA-1 GGTGACGAAGTCTAATCAGG and Il21-sg-RNA-2 GCTGTTCAACAATGTCAATA. Scrambled mouse sgRNA served as control: GCGAGGTATTCGGCTCCGCG. Total BM was isolated from the femur and tibia of 6- to 10-week-old female C57BL/6 WT mice or $B e c n 1^{4 M}$ mice. BM was subjected to erythrocyte lysis (BD PharmLyse, BD Biosciences), followed by magnetic bead selection of cKit-positive cells using CD117 MicroBeads (Miltenyi Biotec). Prior to flow sorting using BD FACSAria II, CD117-selected cells were stained with APC-labeled cKit (eBioscience), PE-labeled Sca1 (BioLegend), Pacific blue-labeled Gr1, CD11b, B220 (CD45R), Ter119, and CD3 (eBiosciences). LSK cells were cultured in StemPro-34 SFM complete medium (Thermo Fisher) supplemented with $50 \mathrm{ng} / \mathrm{mL}$ murine Thpo and 50 ng murine Scf (Life Technologies) for 48 hours and then transduced with concentrated lentiviral supernatant in the presence of $2 \mu \mathrm{g} / \mathrm{mL}$ polybrene. At 24 hours after transduction, cells were collected and i.v. injected into lethally irradiated (950 cGy) 6- to 10-week-old female C57BL/6 mice (Jackson Laboratory). Since the CRISPR-Cas9 genotyping data provided only a "snapshot" of the polyclonal cell population genotype, the possibility that not all alleles had been targeted by this approach could not be ruled out. For BM chimeras, approximately 1.5 $\times 10^{6}$ total BM cells from CD $45.1^{+}$donors (see Supplemental Table 7) were mixed with $1.5 \times 10^{6}$ total BM cells from adult WT (CD45.2 $)$ or Becn $1^{1 M}\left(\mathrm{CD} 45.2^{+}\right)$mice and then were transferred into adult WT CD 45.1 $1^{+}$mice that had been exposed to $950 \mathrm{cGy}$. Chimeras were analyzed at least 6 weeks after reconstitution. To study in vivo endotoxicity, mice received i.p. injections of LPS (25-30 mg/kg) and blood samples were collected to assay plasma cytokine concentrations.

RNA-Seq. Total RNA was used for mRNA isolation and cDNA library generation with the miRNeasy Micro Kit (QIAGEN). On-column DNase digestion (DNase-Free DNase Set, QIAGEN) was performed according to manufacturer protocols to prevent genomic DNA contamination. RNA and library preparation integrity were verified using a 2100 BioAnalyzer system (Agilent). Ribosomal RNA depletion was performed following the low-input protocol of RiboMinus Eukaryote System, version 2 (Thermo Fisher), with 500 ng total RNA input. Finally, libraries were prepared using the Ion Total RNA-Seq Kit, version 2 
(Thermo Fisher), with minor changes to the standard protocol. These included lower amplification cycles and the usage of entire fragmented RNA for cDNA synthesis. For the sequencing reactions, an Ion Torrent Proton platform with V3 chemistry (Ion PI Template OT2 200 Kit, version 3, Thermo Fisher) and PIV2 Chips (Ion PI Chip Kit, version 2, Thermo Fisher) were used. The experiment was performed on 2 chips containing 2 biological replicates per condition (wt1/mt1 on chip A and $w t 2 / \mathrm{mt} 2$ on chip B), resulting in 163 million total reads and at least 40 million reads per library. Base calling was performed on Ion Torrent suite software. Raw sequencing reads in FASTQ format were aligned to the UCSC mouse reference genome (mm10) using TOPHAT21 (58). Differentially expressed genes were identified using DESeq2, version 1.62. Only genes with a minimum $\log _{2}$ fold change of \pm 2 , a maximum Benjamini-Hochberg corrected $P$ value of 0.05 , and a minimum combined mean of 5 reads were classified as having significant differential expression. Gene set enrichment analysis (GSEA) software was used to perform GSEA against the GSEA database (59). DAVID/EASE software (https://david.ncifcrf.gov/summary.jsp) was used to establish whether specific cell functions and biologic processes, defined according to gene ontology, were significantly represented among the deregulated genes. Data were also analyzed with Ingenuity Pathway Analysis (IPA) software (QIAGEN) (see Supplemental Tables 2-4).

Isolation of immune cells. BM cells were isolated from the tibia and femur and cultured in RPMI 1640 medium with 10\% FBS, 1\% penicillinstreptomycin, and $55 \mathrm{mM} \beta$-mercaptoethanol. For BM-derived macrophages (BMDMs), BM cells were cultured in 10\% L929 conditioned media containing macrophage-colony stimulating factor (M-CSF) for 5 days. For BM-derived cDCs, BM cells were cultured in the presence of $20 \mathrm{ng} / \mathrm{mL}$ murine GM-CSF and $10 \mathrm{ng} / \mathrm{ml} \mathrm{IL}-4$ for 6-8 days. Peritoneal macrophages were obtained by injecting mice i.p. with $3 \mathrm{ml} 4 \%(\mathrm{v} / \mathrm{v})$ thioglycollate (BD), and peritoneal cavities were flushed after 3 days with RPMI 1640 media with 2\% FBS. Mouse CD4 cells were isolated from SP or LN by using the Dynabeads Untouched Mouse CD4 Cells Kit (Thermo Fisher). $10^{8}$ cells $/ \mathrm{mL}$ of splenocytes and LN cells were blocked with heat-inactivated FBS and antibody mix against CD8, B220, CD11b, Ter-119, and CD16/32. After 20 minutes incubation at $4^{\circ} \mathrm{C}$, cells were washed with isolation buffer ( $\mathrm{PBS}\left[\mathrm{Ca}^{2+}\right.$ and $\mathrm{Mg}^{2+}$ free] supplemented with $0.1 \%$ BSA and $2 \mathrm{mM}$ EDTA) and incubated with prewashed mouse depletion Dynabeads for 15 minutes at room temperature (RT). Beadbound cells were resuspended by gently pipetting and were placed in the magnet for 2 minutes. Untouched $\mathrm{CD}^{+}{ }^{+} \mathrm{T}$ cells in the supernatant were then collected. Similarly, total B cells were isolated from the tumor, LN, SP, or BM using mouse CD45R (B220) microbeads (Miltenyi Biotec) or PE-positive selection beads with PE-B220 antibody. In some experiments, B cells were pretreated with $1 \mu \mathrm{g} / \mathrm{mL}$ CD $40 \mathrm{~L}$ (Thermo Fisher, catalog 34-8512-80), or anti-CD40 (CD40 agonist) (Thermo Fisher, clone HM40-3, catalog 14-0402-82), 10 ng/mL IL-4 (Thermo Fisher, catalog PMC0045), 100 ng/mL LPS (Sigma-Aldrich, catalog L4391), specific inhibitor of JNK (Sigma-Aldrich, catalog S5567, SP 600125, $5 \mu \mathrm{mol} / \mathrm{L}$ ), ERK (Sigma-Aldrich, catalog 19-147, U0126, $20 \mu \mathrm{mol} / \mathrm{L}$ ), NF-кB (Sigma-Aldrich, catalog B5556, BAY 11-7082, $5 \mu \mathrm{mol} / \mathrm{L}$ ), p38 (Sigma-Aldrich, catalog S8307, SB 203580, $20 \mu \mathrm{mol} / \mathrm{L}$ ), and STAT3 (Sigma-Aldrich, catalog 573097, WP1066, 5 mol/L). Peripheral neutrophils were obtained from the peritoneal cavity by i.p. injection of mice with $2 \mathrm{ml} \mathrm{4 \%} \mathrm{(v/v)} \mathrm{thioglycollate} \mathrm{for} 4$ hours, followed by the collection of peritoneal exudates with RPMI 1640 media with 2\% FBS. Neutrophils were then purified by sorting Ly $6 \mathrm{G}^{+} \mathrm{CD} 11 \mathrm{~b}^{+}$cells or using EasySep
Mouse Neutrophil Enrichment Kit (Stem Cell Technologies) to remove nonneutrophil populations. Tissue-infiltrated neutrophils were purified from intraparenchymal pulmonary or LN cell suspensions using an isolation method similar to that used for peritoneal neutrophils. Briefly, for lung neutrophil isolation, the chest of the mouse was opened, and the lung vascular bed was flushed with $2-3 \mathrm{ml}$ of prechilled PBS injected into the right ventricle. Lungs were excised to avoid the paratracheal LNs and thymus and washed twice in RPMI 1640 media supplemented with penicillin/streptomycin. The excised lungs were minced finely, and the tissue pieces were placed in RPMI 1640 medium containing 2\% FBS, $20 \mathrm{U} / \mathrm{mL}$ collagenase, and $1 \mathrm{~g} / \mathrm{mL}$ DNase. Following incubation for 60 minutes at $37^{\circ} \mathrm{C}$, any remaining intact tissue was disrupted by passage through a 21-gauge needle. Tissue fragments and the majority of dead cells were removed by rapid filtration through a glass-wool column, and cells were collected by centrifugation for 5 minutes at $500 \mathrm{~g}$. Cells were subjected to enrichment using the EasySep Mouse Neutrophil Enrichment Kit.

Immunoprecipitation and immunoblot analyses. For immunoprecipitation, whole-cell lysates were incubated overnight with indicated antibodies plus protein A and $\mathrm{G}$ beads (Pierce). For immunoprecipitation with anti-FLAG, anti-FLAG agarose gels were used. Beads were then washed 5 times with low-salt lysis buffer (50 mM HEPES, $15 \mathrm{mM}$ $\mathrm{NaCl}, 1 \mathrm{mM}$ EDTA, $1.5 \mathrm{mM} \mathrm{MgCl}_{2}, 10 \%$ glycerol, $1 \%$ Triton X-100), and immunoprecipitates were eluted with $\times 4$ SDS loading buffer. Immunoblotting was performed by resolving protein lysates on SDS-PAGE gels, followed by transfer to nitrocellulose membranes (Bio-Rad) and further incubation of membranes with indicated antibodies overnight (see Supplemental Table 7). For all blots, EMD Millipore Luminata Western HRP Chemiluminescence Substrate was used for protein detection (see complete unedited blots in the supplemental material).

Flow cytometry. Single-cell suspensions were obtained from tissues and stained for 30 minutes with indicated antibodies. For intracellular staining, $\mathrm{CD}^{+}$or $\mathrm{CD} 8^{+} \mathrm{T}$ cells were stimulated with PMA $(15 \mathrm{nM})$ and ionomycin $(0.5 \mu \mathrm{M})$ for 6 hours at $37^{\circ} \mathrm{C}$ in the presence of GolgiStop (monensin) (BD Biosciences - Pharmingen). Cells were then stained with the surface marker (see Supplemental Table 7) for 15 minutes on ice and permeabilized using Cytofix/Cytoperm (BD Biosciences) for 30 minutes on ice. Permeabilized cells were resuspended in BD Perm/ Wash buffer (BD Biosciences) and stained with cytokine antibody for 20 minutes. FACS analysis was performed with BD LSRII Flow Cytometer (BD), and data were analyzed by BD FACSDiva software.

Cytokine release assay. Cell supernatants or sera were collected at indicated time points after stimulation with LPS with or without ATP. Cytokine concentrations were determined by ELISA with TNF- $\alpha$, IL-6, IL-1 $\beta$, IL-17A, IL-17F, IL-21, IL-22, and BAFF antibodies (see Supplemental Table 7). ELISAs were performed according to the manufacturer's instructions. In brief, 96-well plates were precoated with the capture antibody ( $1: 500$ in coating buffer) at $4^{\circ} \mathrm{C}$ overnight. On the next day, the plate was washed with PBS/0.1\% Tween 20 and blocked with 1\%BSA/PBS diluent buffer for 2 hours at RT. Diluted supernatants and cytokine standards were then applied to the plate and incubated for 2 hours at RT. The plate was then washed and incubated with biotin-conjugated detection antibody (1:1000 in 1\%BSA/PBS diluent buffer) for 1 hour at RT. The plate was then washed and incubated with poly-HRP streptavidin (1:5000 in the diluent buffer, Thermo Scientific) for 30 minutes. The plate was washed again and finally incubated with the tetramethylbenzidine substrate solution (Sigma-Aldrich). The reaction 
was stopped using $2 \mathrm{M} \mathrm{H}_{2} \mathrm{SO}_{4}$. The absorbance of each well was recorded by a Synergy 2 plate reader (BioTek) at $450 \mathrm{~nm}$. The absorbance of the standard sample was used to construct the standard curve.

Hematological and histological analysis. Complete peripheral blood counts were performed with an automated hematology analyzer (Hemavet 850; Drew Scientific). For whole BM sections, specimens were decalcified in (8\%) hydrochloric acid/(8\%) formic acid working solution overnight, followed by neutralization by ammonia solution for 30 minutes, and were embedded in paraffin. Embedded BM blocks were sliced and stained with H\&E for conventional morphological assessment. For cytocentrifugation preparations, BM cells were subjected to red blood lysis in ACK buffer $\left(0.15 \mathrm{mM} \mathrm{NH}_{4} \mathrm{Cl}, 1 \mathrm{mM} \mathrm{KHCO}_{3}\right.$, and $0.1 \mathrm{mM} \mathrm{Na}{ }_{2}$ EDTA). Between 2 and $5 \times 10^{5}$ cells were suspended in $100 \mu \mathrm{L}$ PBS, loaded onto disposable Cytofunnel chambers, and centrifuged for 4 minutes at $500 \mathrm{~g}$ using a CytoSpin III cytocentrifuge (Shandon; Thermo Fisher Scientific). For May-Grünwald Giemsa stain, cytocentrifuged samples were stained in May-Grünwald solution (MG500; Sigma-Aldrich) for 2 minutes and Giemsa (GS500, 5\% solution in water; Sigma-Aldrich) for 12 minutes, followed by 2 washes in distilled water for 30 seconds. Thereafter, samples were cytocentrifuged, counterstained with May-Grünwald solution for 10 seconds, and washed in water. Slides were coverslipped using Permount and analyzed by brightfield microscopy. IHC staining was performed with the streptavidin-biotin-peroxidase complex method using the Vectastain Elite ABC Kit and the DAB Peroxidase (HRP) Substrate Kit (Vector Laboratories). Primary antibodies used are listed in Supplemental Table 7. Negative control staining was performed using mouse, rabbit, or rat immune sera instead of the primary antibodies. Sections of human B cell lymphoblastic leukemia/lymphoma tissues were obtained in-house, including 10 pre-B ALLs in LN or BM and 6 reactive LNs or BM controls. IF assays and confocal microscopy were conducted using fluorescent secondary antibodies (see Supplemental Table 7). Samples were visualized using a Nikon Eclipse Ti-E microscope. All acquired images were analyzed using the Nikon NIS-Elements AR package or ImageJ software (NIH).

In vivo cell depletion and antibody blockade. Monocyte or macrophage depletion was performed by i.v. injection of $250 \mu \mathrm{l}$ clodronate-containing liposomes (Encapsula NanoSciences) on day -1 relative to LPS treatment. Control liposomes were used as PBS control. Neutrophil/granulocyte depletion was induced with combined $0.5 \mathrm{mg}$ of anti-Ly6 $\mathrm{G} \mathrm{mAb}$ clone 1A8 (BioXcell) or with i.p. injection of PBS controls into mice on days $-3,-2$, and -1 relative to LPS treatment. FACS-sorted $\mathrm{B}_{2} 2 \mathrm{O}^{+}$cells were left untreated or were cocultured with isolated neutrophils in the presence or absence of $10 \mu \mathrm{g} / \mathrm{mL}$ antibodies against IL-21, BAFF, IFN- $\gamma$, IL-1 $\beta$, or CD40L (see Supplemental Table 7).

Quantitative RT-PCR analysis. Total RNA was isolated from cells using RNeasy RNA isolation kit (QIAGEN) and reverse transcribed using SuperScript III Reverse Transcriptase (Invitrogen) per the manufacturer's directions. Quantitative PCR was conducted on the ABI Prism 7000 analyzer (Applied Biosystems) using SYBR GreenER qPCR Super Mix Universal (Invitrogen). mRNA levels were normalized to Gapdh, and relative levels were determined using the $\triangle \Delta \mathrm{CT}$ method. Primers are listed in Supplemental Table 6.

ROS measurement and $\mathrm{H}_{2} \mathrm{O}_{2}$ treatment. Neutrophils were stimulated with LPS (100 ng/mL) for 0 minutes, 10 minutes, 30 minutes, and 3 hours at $37^{\circ} \mathrm{C}$. Cells were then incubated with or without CM-H2DCFDA $(10 \mu \mathrm{M})$ in PBS for 30 minutes at $37^{\circ} \mathrm{C}$ and analyzed by flow cytometry using the FITC channel.
Proliferation and apoptosis assays. Annexin V and 7AAD/PI staining kit (BD Biosciences) were used to detect apoptotic cells following the manufacturer's instructions. T cell proliferation was assessed with $\left[{ }^{3} \mathrm{H}\right]$ thymidine assay using irradiated neutrophils as APCs. Briefly, $\mathrm{T}$ cells $\left(1 \times 10^{5}\right)$ were isolated from SPs and cocultured with irradiated Ly $6 \mathrm{G}^{+} \mathrm{CD} 11 \mathrm{~b}^{+}$cells $\left(2 \times 10^{5}\right)$ with the anti-CD3 antibody for 56 hours in 96-well plates. $\mathrm{T}$ cell proliferation was determined by labeling cultured cells with $\left[{ }^{3} \mathrm{H}\right]$ thymidine for 16 hours, and radioactivity was measured using a scintillation counter. CFSE staining was performed by labeling $B$ cells $\left(1 \times 10^{6}\right)$ with CFSE $(5 \mu \mathrm{M})$ for 10 minutes. Cells were washed with PBS, plated in RPMI media containing $10 \%$ FBS and $1 \%(\mathrm{v} / \mathrm{v})$ penicillin-streptomycin supplemented with LPS (100 ng/mL) and IL-4 (50 ng/ $\mathrm{mL}$ ), and incubated at $37^{\circ} \mathrm{C}$ until they were analyzed by flow cytometry.

GEP of human lymphomas. Gene expression analysis was performed on data sets from the NCI's Gene Expression Omnibus database (GEO GSE12195, GSE26673, GSE16455, GSE12195, GSE16455, GSE7440, GSE11877). Specifically, we included the following cancer types: 16 Burkitt lymphoma (BL), 18 CLL, 38 follicular lymphoma (FL), 75 DLBCL, 22 MCL, 4 marginal zone lymphoma (MZL), and 306 B-cell ALL, and 5 naive cell samples. Data were processed by Affymetrix Expression Console (see Supplemental Table 5). Statistical differences among multiple groups were analyzed with 1-way ANOVA. Significance was determined as a $P$ value of less than 0.05. All gene expression data for ALL, which derived from pre-B cells, are obtained from TARGET (https://ocg.cancer.gov/programs/target/data-matrix), including phase I and phase II (B-cell ALL) studies of approximately 200 relapse-enriched cases (patients $>9$ years old, without BCR/ABL fusion and hypodiploid), with an additional 500 pre-B cell all cases for validation in a phase II study $(60,61)$. Gene expression data were processed using the Affymetrix U133 Plus 2.0 Array platform. For the correlation between transcriptional expression of IL-21, CD274, CD177, and LY6G6D, we used Spearman's correlation and considered $|\mathrm{Rs}|>$ 0.2 and $\mathrm{FDR}<0.05$ as significant correlations.

Statistical analysis. Data were analyzed by Student's 2-tailed $t$ test or 1-way ANOVA for multiple comparisons or Mantel-Cox log-rank test for mouse survival using GraphPad Prism 4.0 software and represented as mean \pm SEM. Differences were considered significant at $P<0.05$. The correlation of transcriptional expression was calculated by Spearman's correlation, and $|\mathrm{R}|>0.2$ and $\mathrm{FDR}<0.05$ were considered significant.

Study approval. Animal experiments in this study were approved and carried out following the protocol (AUP-0115-0005) provided by the IACUC at Houston Methodist Research Institute. IACUC uses the NIH Guide for the Care and Use of Laboratory Animals, which is based on the US Government Principles for Utilization and Care of Vertebrate Animals Used in Testing, Research, and Training. The human subject study was performed in accordance with institutional guidelines and the protocol approved by the Institutional Review Board of Houston Methodist Research Institute (IBC00000357). All subjects gave informed consent.

Data availability. Data supporting the findings of this study are available from the corresponding author upon reasonable request. Source data for RNA-Seq were deposited in the NCBI's GEO database (GEO GSE132899).

\section{Author contributions}

RFW supervised the entire project. PT designed, performed, and analyzed experiments and data. L He, CX, JM, XY, MZ, YZ, and 
HYW provided input and technical support. LD and L Han performed human patient database analysis. JMY provided human patient samples and performed pathological analysis. PT and RFW wrote the manuscript.

\section{Acknowledgments}

This work was supported in part by grants from the NIH (R01CA101795), the Cancer Prevention and Research Institute of
Texas (RP170537), the US Department of Defense (DoD) Breast Cancer Research Program (BCRP) (BC151081), and Golfers Against Cancer (to RFW). The funders had no role in study design, data collection and analysis, decision to publish, or preparation of the manuscript.

Address correspondence to: Rong-Fu Wang, 6670 Bertner Ave, R9-460, Houston, Texas 77030, USA. Phone: 713.441.7359; Email: rwang3@houstonmethodist.org.
1. Lorin S, Hamaï A, Mehrpour M, Codogno P. Autophagy regulation and its role in cancer. Semin Cancer Biol. 2013;23(5):361-379.

2. Deretic V, Saitoh T, Akira S. Autophagy in infection, inflammation and immunity. Nat Rev Immunol. 2013;13(10):722-737.

3. Qu X, et al. Promotion of tumorigenesis by heterozygous disruption of the beclin 1 autophagy gene. J Clin Invest. 2003;112(12):1809-1820.

4. Powell DR, Huttenlocher A. Neutrophils in the tumor microenvironment. Trends Immunol. 2016;37(1):41-52.

5. Zenaro E, et al. Neutrophils promote Alzheimer's disease-like pathology and cognitive decline via LFA-1 integrin. Nat Med. 2015;21(8):880-886.

6. Gätjen M, et al. Splenic marginal zone granulocytes acquire an accentuated neutrophil B-cell helper phenotype in chronic lymphocytic leukemia. Cancer Res. 2016;76(18):5253-5265.

7. Sangaletti S, et al. Defective stromal remodeling and neutrophil extracellular traps in lymphoid tissues favor the transition from autoimmunity to lymphoma. Cancer Discov. 2014;4(1):110-129.

8. Puga I, et al. B cell-helper neutrophils stimulate the diversification and production of immunoglobulin in the marginal zone of the spleen. Nat Immunol. 2011;13(2):170-180.

9. Sun C, Mezzadra R, Schumacher TN. Regulation and function of the PD-L1 checkpoint. Immunity. 2018;48(3):434-452.

10. Goodman A, Patel SP, Kurzrock R. PD-1-PD-L1 immune-checkpoint blockade in B-cell lymphomas. Nat Rev Clin Oncol. 2017;14(4):203-220.

11. Pfeiler S, et al. Distinct surveillance pathway for immunopathology during acute infection via autophagy and SR-BI. Sci Rep. 2016;6:34440.

12. Mittal M, Siddiqui MR, Tran K, Reddy SP, Malik AB. Reactive oxygen species in inflammation and tissue injury. Antioxid Redox Signal. 2014;20(7):1126-1167.

13. van Rooijen N, Bakker J, Sanders A. Transient suppression of macrophage functions by liposome-encapsulated drugs. Trends Biotechnol. 1997;15(5):178-185.

14. Vassiloyanakopoulos AP, Okamoto S, Fierer $\mathrm{J}$. The crucial role of polymorphonuclear leukocytes in resistance to Salmonella dublin infections in genetically susceptible and resistant mice. Proc Natl Acad Sci USA. 1998;95(13):7676-7681.

15. Li TJ, et al. Interleukin-17-producing neutrophils link inflammatory stimuli to disease progression by promoting angiogenesis in gastric cancer. Clin Cancer Res. 2017;23(6):1575-1585.

16. Li L, et al. IL-17 produced by neutrophils regulates IFN-gamma-mediated neutrophil migration in mouse kidney ischemia-reperfusion injury. JClin Invest. 2010;120(1):331-342.

17. Taylor PR, et al. Activation of neutrophils by autocrine IL-17A-IL-17RC interactions during fungal infection is regulated by IL-6, IL-23, ROR $\gamma \mathrm{t}$ and dectin-2. Nat Immunol. 2014;15(2):143-151.

18. Arthur JS, Ley SC. Mitogen-activated protein kinases in innate immunity. Nat Rev Immunol. 2013;13(9):679-692.

19. Xu P, et al. Quantitative proteomics reveals the function of unconventional ubiquitin chains in proteasomal degradation. Cell. 2009;137(1):133-145.

20. Heckl D, et al. Generation of mouse models of myeloid malignancy with combinatorial genetic lesions using CRISPR-Cas9 genome editing. Nat Biotechnol. 2014;32(9):941-946.

21. Morse HC, et al. Bethesda proposals for classification of lymphoid neoplasms in mice. Blood. 2002;100(1):246-258.

22. Stranges $\mathrm{PB}$, et al. Elimination of antigen-presenting cells and autoreactive $T$ cells by Fas contributes to prevention of autoimmunity. Immunity. 2007;26(5):629-641.

23. Nie Y, Waite J, Brewer F, Sunshine MJ, Littman DR, Zou YR. The role of CXCR4 in maintaining peripheral B cell compartments and humoral immunity. JExp Med. 2004;200(9):1145-1156.

24. Allende ML, Tuymetova G, Lee BG, Bonifacino E, Wu YP, Proia RL. S1P1 receptor directs the release of immature $B$ cells from bone marrow into blood. J Exp Med. 2010;207(5):1113-1124.

25. Somasundaram R, Prasad MA, Ungerbäck J, Sigvardsson M. Transcription factor networks in B-cell differentiation link development to acute lymphoid leukemia. Blood. 2015;126(2):144-152.

26. Shojaee S, et al. PTEN opposes negative selection and enables oncogenic transformation of pre-B cells. Nat Med. 2016;22(4):379-387.

27. Liu H, et al. Nuclear cGAS suppresses DNA repair and promotes tumorigenesis. Nature. 2018;563(7729):131-136.

28. Rickert RC. New insights into pre-BCR and BCR signalling with relevance to $\mathrm{B}$ cell malignancies. Nat Rev Immunol. 2013;13(8):578-591.

29. Yu H, Pardoll D, Jove R. STATs in cancer inflammation and immunity: a leading role for STAT3. Nat Rev Cancer. 2009;9(11):798-809.

30. Malin S, et al. Role of STAT5 in controlling cell survival and immunoglobulin gene recombination during pro-B cell development. Nat Immunol. 2010;11(2):171-179.

31. Jarosinski KW, Massa PT. Interferon regulatory factor-1 is required for interferon-gammainduced MHC class I genes in astrocytes. JNeuroimmunol. 2002;122(1-2):74-84.
32. Garcia-Diaz A, et al. Interferon receptor signaling pathways regulating PD-L1 and PD-L2 expression. Cell Rep. 2017;19(6):1189-1201.

33. Coelho MA, et al. Oncogenic RAS signaling promotes tumor immunoresistance by stabilizing PD-L1 mRNA. Immunity. 2017; 47(6):1083-1099.e6.

34. Mezzadra R, et al. Identification of CMTM6 and CMTM4 as PD-L1 protein regulators. Nature. 2017;549(7670):106-110.

35. Loughner CL, Bruford EA, McAndrews MS, Delp EE, Swamynathan S, Swamynathan SK. Organization, evolution and functions of the human and mouse Ly6/uPAR family genes. Hum Genomics. 2016;10:10.

36. Lee PY, Wang JX, Parisini E, Dascher CC, Nigrovic PA. Ly6 family proteins in neutrophil biology. J Leukoc Biol. 2013;94(4):585-594.

37. Martinez J, et al. Molecular characterization of LC3-associated phagocytosis reveals distinct roles for Rubicon, NOX2 and autophagy proteins. Nat Cell Biol. 2015;17(7):893-906.

38. Gutierrez MG, Master SS, Singh SB, Taylor GA, Colombo MI, Deretic V. Autophagy is a defense mechanism inhibiting BCG and Mycobacterium tuberculosis survival in infected macrophages. Cell. 2004;119(6):753-766.

39. Baldassare JJ, Bi Y, Bellone CJ. The role of p38 mitogen-activated protein kinase in IL-1 beta transcription. J Immunol. 1999;162(9):5367-5373.

40. Noubade R, et al. Activation of p 38 MAPK in CD4 T cells controls IL-17 production and autoimmune encephalomyelitis. Blood. 2011;118(12):3290-3300.

41. Greten FR, et al. NF-kappaB is a negative regulator of IL-1beta secretion as revealed by genetic and pharmacological inhibition of IKKbeta. Cell. 2007;130(5):918-931.

42. Hsu LC, et al. IL-1 $\beta$-driven neutrophilia preserves antibacterial defense in the absence of the kinase IKK $\beta$. Nat Immunol. 2011;12(2):144-150.

43. Aras S, Zaidi MR. TAMeless traitors: macrophages in cancer progression and metastasis. $\mathrm{Br} \mathrm{J}$ Cancer. 2017;117(11):1583-1591.

44. Mueller SN, Germain RN. Stromal cell contributions to the homeostasis and functionality of the immune system. Nat Rev Immunol. 2009;9(9):618-629.

45. Hendriks RW, Yuvaraj S, Kil LP. Targeting Bruton's tyrosine kinase in B cell malignancies. Nat Rev Cancer. 2014;14(4):219-232.

46. Beck TC, Gomes AC, Cyster JG, Pereira JP. CXCR4 and a cell-extrinsic mechanism control immature B lymphocyte egress from bone marrow. J Exp Med. 2014;211(13):2567-2581.

47. Cyster JG, Schwab SR. Sphingosine-1-phosphate 
and lymphocyte egress from lymphoid organs. Annu Rev Immunol. 2012;30:69-94.

48. Le Y, et al. SOCS3 protein developmentally regulates the chemokine receptor CXCR4-FAK signaling pathway during B lymphopoiesis. Immunity. 2007;27(5):811-823.

49. Yoshida N, et al. CXCR4 expression on activated $\mathrm{B}$ cells is downregulated by CD63 and IL-21. JImmunol. 2011;186(5):2800-2808.

50. Bubier JA, et al. A critical role for IL-21 receptor signaling in the pathogenesis of systemic lupus erythematosus in BXSB-Yaa mice. Proc Natl Acad Sci USA. 2009;106(5):1518-1523.

51. Stolfi C, Pallone F, Macdonald TT, Monteleone G. Interleukin-21 in cancer immunotherapy: Friend or foe? Oncoimmunology. 2012;1(3):351-354.
52. Ware CF. APRIL and BAFF connect autoimmunity and cancer. J Exp Med. 2000;192(11):F35-F38.

53. Scott DW, Gascoyne RD. The tumour microenvironment in B cell lymphomas. Nat Rev Cancer. 2014;14(8):517-534.

54. Leonard WJ, Wan CK. IL-21 signaling in immunity. F100ORes. 2016;5:F1000 Faculty Rev-224.

55. Kinter AL, et al. The common gamma-chain cytokines IL-2, IL-7, IL-15, and IL-21 induce the expression of programmed death- 1 and its ligands. J Immunol. 2008;181(10):6738-6746.

56. Zou W, Wolchok JD, Chen L. PD-L1 (B7-H1) and PD-1 pathway blockade for cancer therapy: mechanisms, response biomarkers, and combinations. Sci Transl Med. 2016;8(328):328rv4.

57. Shalapour S, et al. Inflammation-induced IgA+ cells dismantle anti-liver cancer immunity. Nature. 2017;551(7680):340-345.

58. Trapnell C, Pachter L, Salzberg SL. TopHat: discovering splice junctions with RNA-Seq. Bioinformatics. 2009;25(9):1105-1111.

59. Subramanian A, et al. Gene set enrichment analysis: a knowledge-based approach for interpreting genome-wide expression profiles. Proc Natl Acad Sci USA. 2005;102(43):15545-15550.

60. Loh ML, et al. Tyrosine kinome sequencing of pediatric acute lymphoblastic leukemia: a report from the Children's Oncology Group TARGET Project. Blood. 2013;121(3):485-488.

61. Ma X, et al. Rise and fall of subclones from diagnosis to relapse in pediatric B-acute lymphoblastic leukaemia. Nat Commun. 2015;6:6604. 